\title{
Boundary-Layer-Ingesting Inlet Flow Control
}

\author{
Lewis R. Owens ${ }^{1}$, Brian G. Allan ${ }^{2}$ and Susan A. Gorton ${ }^{3}$ \\ NASA Langley Research Center, Hampton, VA 23681
}

\begin{abstract}
This paper gives an overview of a research study conducted in support of the small-scale demonstration of an active flow control system for a boundary-layer-ingesting (BLI) inlet. The effectiveness of active flow control in reducing engine inlet circumferential distortion was assessed using a $2.5 \%$ scale model of a $35 \%$ boundary-layer-ingesting flush-mounted, offset, diffusing inlet. This experiment was conducted in the NASA Langley 0.3-meter Transonic Cryogenic Tunnel at flight Mach numbers with a model inlet specifically designed for this type of testing. High mass flow actuators controlled the flow through distributed control jets providing the active flow control. A vortex generator point design configuration was also tested for comparison purposes and to provide a means to examine a hybrid vortex generator and control jets configuration. Measurements were made of the onset boundary layer, the duct surface static pressures, and the mass flow through the duct and the actuators. The distortion and pressure recovery were determined by 40 total pressure measurements on 8 rake arms each separated by 45 degrees and were located at the aerodynamic interface plane. The test matrix was limited to a maximum free-stream Mach number of 0.85 with scaled mass flows through the inlet for that condition. The data show that the flow control jets alone can reduce circumferential distortion $\left(\mathrm{DPCP}_{\text {avg }}\right)$ from 0.055 to about 0.015 using about $2.5 \%$ of inlet mass flow. The vortex generators also reduced the circumferential distortion from 0.055 to 0.010 near the inlet mass flow design point. Lower inlet mass flow settings with the vortex generator configuration produced higher distortion levels that were reduced to acceptable levels using a hybrid vortex generator/control jets configuration that required less than $1 \%$ of the inlet mass flow.
\end{abstract}

\section{Nomenclature}

$\mathrm{D}_{2}$

h

$\mathrm{H}$

$\mathrm{H}_{\mathrm{i}}$

$\mathrm{H}_{\max }$

distance between the inlet lip highlight station and the inlet throat station (see fig. 3 ), in.

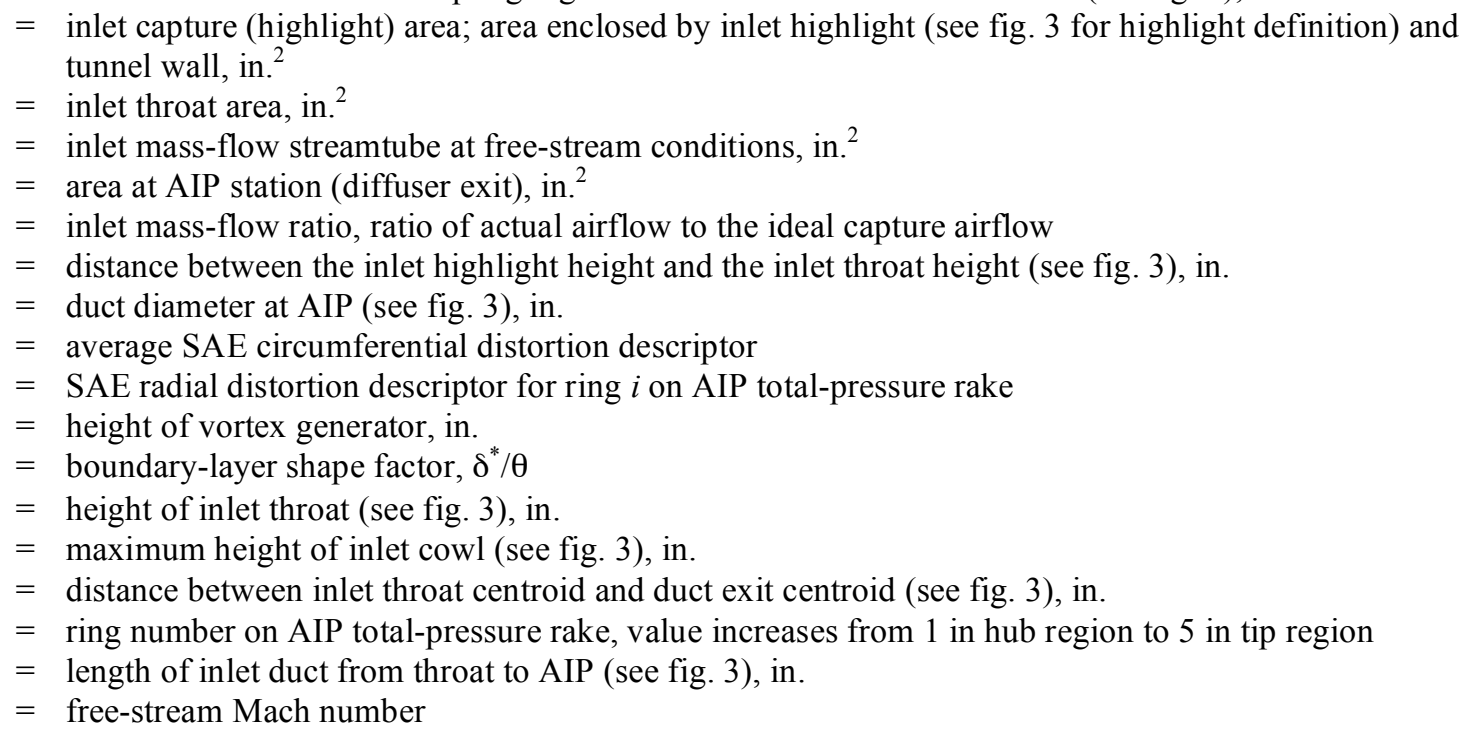

\footnotetext{
${ }^{1}$ Research Engineer, Flow Physics and Control Branch, MS 170, NASA Langley Research Center, Hampton, VA 23681, Senior Member.

2 Research Scientist, Flow Physics and Control Branch, MS 170, NASA Langley Research Center, Hampton, VA 23681, Senior Member.

3 Branch Head, Flow Physics and Control Branch, MS 170, NASA Langley Research Center, Hampton, VA 23681, Member.
} 


$\begin{array}{ll}\mathrm{P}_{\mathrm{t}} & =\text { free-stream total pressure, psi } \\ \mathrm{P}_{\mathrm{t}, 2, \mathrm{avg}} & =\text { area weighted average total pressure at AIP } \\ \mathrm{P}_{\mathrm{t}, 2, \mathrm{avg}} / \mathrm{P}_{\mathrm{t}} & =\text { inlet recovery pressure ratio } \\ \mathrm{Rn}_{\mathrm{ft}} & =\text { Reynolds number per foot, } 1 / \mathrm{ft} \\ \mathrm{Re}_{\mathrm{D}} & =\text { Reynolds number based on duct AIP diameter } \\ \mathrm{T}_{\mathrm{t}} & =\text { free-stream total temperature, }{ }^{\circ} \mathrm{R} \\ \mathrm{U} & =\text { velocity at each boundary-layer rake probe measurement, } \mathrm{ft} / \mathrm{sec} \\ \mathrm{U}_{\mathrm{e}} & =\text { boundary-layer edge velocity, } \mathrm{ft} / \mathrm{sec} \\ \mathrm{U} / \mathrm{U}_{\mathrm{e}} & =\text { boundary-layer profile velocity ratio } \\ \mathrm{W}_{\text {act }} & =\text { airflow rate measured by venturi, lb } \mathrm{b} / \mathrm{sec} \\ \mathrm{W}_{\mathrm{i}} & =\text { inlet throat maximum width (see fig. } 3 \text { ), in. } \\ \mathrm{X}_{\mathrm{aft}} & =\text { cowl aftbody length (see fig. } 3 \text { ), in. } \\ \mathrm{XPLUG} & =\text { position of the inlet mass flow measurement plug, in. } \\ \delta & =\text { measured boundary-layer thickness, in. } \\ \delta^{*} & =\text { measured boundary-layer displacement thickness, in. } \\ \theta & =\text { measured boundary-layer momentum thickness, in. }\end{array}$

Abbreviations:

$\begin{array}{ll}\text { AFC } & \text { active flow control } \\ \text { AIP } & \text { aerodynamic interface plane } \\ \text { BLI } & \text { boundary-layer ingesting } \\ \text { BWB } & \text { blended-wing-body } \\ \text { CFD } & \text { computational fluid dynamics } \\ \text { NASA } & \text { National Aeronautics and Space Administration } \\ \text { SAE } & \text { Society of Automotive Engineers } \\ \text { UEET } & \text { Ultra Efficient Engine Technology } \\ \text { VGs } & \text { vortex generators }\end{array}$

\section{Introduction}

$\mathrm{T}$ HE effect of aviation on the environment and in particular global warming has recently become a focus of study ${ }^{1-2}$ This study considers the three primary impacts that civil aviation has on the environment. These impacts include noise, pollution around airports and emissions at altitude. In response to environmental concerns and to foster revolutionary propulsion technologies, NASA launched the Ultra Efficient Engine Technology (UEET) program in late $1999^{3}$. This program had several elements, one of which was to explore the feasibility of the Blended-Wing-Body (BWB) concept as an efficient alternative to conventional transport configurations. The BWB concept has been considered in various forms for several years. ${ }^{4-7}$ System studies have shown that in order to make the largest impact on the vehicle performance, the engines and inlets should be placed near the upper surface on the aft section of the vehicle. However, the incorporation of the inlets on the surface of the vehicle increases the technical risk of the configuration. ${ }^{6}$ Although Boeing reduced this risk by positioning the engines on pods for most of their configuration studies, the NASA UEET program has continued to pursue the goal of boundary-layeringesting (BLI) flush-mounted, offset inlets for the BWB. Additional system studies, sponsored by NASA, continue to indicate the additional advantages of BLI inlet for the configuration including less fuel burn and lower noise characteristics. The configuration of the BWB with BLI engine inlets is shown in Refs. 6 and 8 and pictured in Figure 1.

When the engines are positioned near the surface, the BWB engine inlet will likely be an S-duct inlet with the capability to ingest the large boundary layer that will build up over the aircraft body. The inlet must perform this task in a manner compatible with engine performance requirements for distortion and pressure recovery. Since the boundary layer on the BWB is expected to be on the order of $30 \%$ of the inlet height, this presents a challenging task for inlet design. In addition, the performance assessment of such a highly-integrated propulsion system is a complex undertaking, requiring the simultaneous examination of many influential factors in order to determine whether BLI provides a benefit from a system standpoint. The trade-offs among reduced drag, weight savings or penalty, and engine operation must all be considered to assess the relative benefit of BLI technology. However, the engine must be able to operate acceptably in the BLI environment. Therefore, an acceptable level of distortion for engine operation must be achieved even if the engine operates at reduced efficiency levels. 
This requirement for at least a minimum level of inlet performance under the severe conditions of an adverse pressure gradient from the S-duct and a very large onset boundary layer flow have led to the consideration of flow control devices in the inlet to control the flow in this type of configuration. Passive flow control in the form of micro-vortex generators or micro-vanes can be used to improve the inlet flow. ${ }^{8-13}$ Reference 8 discusses work using passive devices for an S-duct with BLI while active flow control methods have also been investigated as a means to improve inlet flow for aggressive serpentine inlets with minimal BLI. ${ }^{12-13}$

The effect of BLI on engine performance is known to be detrimental because BLI increases the distortion and reduces the pressure recovery at the inlet exit. ${ }^{14}$ However, the overall system benefits of BLI must be analyzed to determine whether there is a benefit from BLI for a configuration. Reference 6 cites work done early in the development of the BWB configuration that indicated BLI might improve performance as much as $10 \%$. As NASA has continued to explore the benefits of BLI for the BWB, there have been continued efforts to assess the BLI benefits in a progressively higher fidelity manner. As part of the systems benefits assessment, NASA sponsored a contract with The Boeing Company in 2001 to assess the BLI benefits for a representative BWB configuration. The method and findings were presented in reference 15 . The predicted range improvements from a comparison between a flush-mounted BLI inlet and a pylon-mounted inlet from this system study are shown in Figure 2.

The method of analysis used in this study assumes that the application of active flow control will decrease distortion of the inlet to a level that allows acceptable engine operation. Therefore, the method does not assess distortion as one of the factors. This is the underlying assumption that is included in most systems analysis of BLI that presumes that a technology such as active flow control (AFC) will enable operations in a BLI environment. Thus the systems analysis only assesses the effect of BLI; the AFC performance is presumed in the basic analysis.

Much research ${ }^{15-28}$ is already underway to identify and develop active flow control devices and technologies for a variety of applications. This recent emphasis on active flow control and the successful progress in development of actuators, design tools, and control methodologies encouraged the hypothesis that a significant inlet boundary layer could be managed and improved by the application of active flow control. The current NASA study has progressed through four different phases to obtain the results presented in this report. Phase 1 included the development of a new high-Reynolds-number test capability for flush-mounted inlets in the NASA 0.3-meter Transonic Cryogenic Tunnel and the details of this development process are described in references 29 through 31 . Phase 2 included the evaluation and selection of a control jet actuator system from simplified testing of control jets on an adverse pressure gradient ramp. Some of this testing is described more fully in reference 28 , but the results for the final jet actuator selection are unpublished. Phase 3 consisted of the low Mach number $(M=0.15)$ testing of the selected control jet system with the BLI inlet geometry and is described in more detail in reference 15 . The current phase, Phase 4, consisted of the high Mach number $(\mathrm{M}=0.85)$ testing of the selected jet system with the BLI inlet geometry and is described in more detail in this report. The distribution of the control jets in the inlet during this phase of the investigation was guided by the CFD study described in reference 32 .

The purpose of the present investigation was to experimentally demonstrate active flow control at conditions representative of flight Mach numbers and extend the inlet flow control experience beyond the low Mach number demonstration. ${ }^{15}$ During the recent experiment, an inlet with 35\% BLI was tested at a free-stream Mach number of 0.85 over part of the inlet mass flow operational range. High mass flow actuators and micro vortex generators were used to provide flow control inside the inlet. Measurements of inlet distortion and pressure recovery were made at the Aerodynamic Interface Plane (AIP) to provide a means to assess the flow control effectiveness.

\section{Experimental Apparatus and Methods}

\section{A. Facility}

The NASA Langley Research Center 0.3-meter Transonic Cryogenic Tunnel was used in this experiment. ${ }^{33-34}$ The closed-circuit, fan-driven tunnel has a 13- by 13-inch test cross-section with adaptive upper and lower walls. The facility can run in either an air or gaseous nitrogen mode of operation. The tunnel operates with total pressure ranging from 14.7 to 88 psia, Mach numbers ranging from 0.1 to 0.9 , and Reynolds numbers up to 100 million per foot. A high-pressure air supply was used to supply the active flow control jets in the inlet model. The test could not be conducted at cryogenic temperatures because the air supply contained water vapor (dew-point approximately $-40^{\circ} \mathrm{F}$ ), which posed the potential for frosting issues. The primary objective of this test was to examine the flow control effectiveness at high Mach number conditions, so high Reynolds number testing went beyond the scope of the study given the available resources. The testing performed used warm nitrogen gas so that the tunnel pressurization rates would be adequate to balance the venting rates associated with the inlet model in order to 
achieve steady-state test conditions. The tunnel air pressurization system could not balance the given inlet venting rates and was not used during any of the flow control testing.

\section{B. Model}

The model used in this experiment was designed and fabricated for the investigation described in detail in reference 31. The inlet was designed in 2000 by Boeing under contract to NASA to provide an inlet that was representative of a general class of inlets that could be considered for application in a commercial BWB configuration. The definition of inlet parameters and a list of inlet characteristics are given in figure 3 and table 1, respectively. For the inlet flow control investigation, only inlet model A of reference 31 was modified to have a capability to use internal flow control jets. Pictures of the flow control inlet model flush-mounted to the tunnel sidewall are shown in figure 4. On the opposite side of the tunnel wall from where the model was mounted is the plenum area inside the tunnel pressure shell. A view of the plenum side of the tunnel wall with the pressure shell wall removed is presented in figure 5 .

In the center of the picture in figure 5 is a large tubing bundle along with several smaller ones. These tubing bundles are the control jet air supply tubing and the pressure measurement tubing. This is the area where the diffuser passes through the tunnel wall to connect to the instrumentation section at the diffuser exit. This instrumentation section is used to measure the inlet distortion and pressure recovery at the aerodynamic interface plane (AIP), which has a diameter $\left(\mathrm{D}_{2}\right)$ of 2.448 inches. The flow path goes through a $180^{\circ}$-turn to enter the calibrated mass-flow plug assembly. The remote-controlled, mass-flow plug provided both the inlet mass-flow control and the inlet mass-flow rate measurement for this test. The inlet mass-flow rate changes as the position of the plug relative to the bell-mouth is changed. After passing through the mass-flow plug assembly, the flow passes out of the tunnel and is vented to the atmosphere. The pressure difference between the tunnel total pressure and the atmospheric pressure drives the flow through the inlet. For this experiment, the ratio of the tunnel total pressure to the atmospheric pressure was maintained at approximately two, which was sufficient to drive the inlet flow in an acceptable region for operation of the mass-flow plug.

The desired inlet flow operation range is summarized in table 2. The inlet mass flow ratio, $A_{O} / A_{C}$, operational range of interest is 0.46 to 0.65 at the cruise conditions of $\mathrm{M}=0.85$ and a 36,000 feet altitude. The inlet model system used in the experiment could only produce $A_{O} / A_{C}$ levels up to about 0.55 at the tunnel test conditions. This limited inlet mass flow ratio is described in reference 31 as resulting from a problem with underestimating the displacement boundary layer thickness in the inlet design such that the inlet throat was not able to pass the proper amount of flow rate. Pressure measurements inside the diffuser did not reach levels to indicate that the flow inside the inlet was choking. Instead, the flow area of the valve outside the tunnel that vents directly into atmospheric pressure conditions is believed to be the source of choking. There are no specific measurements in this vent valve region to confirm this hypothesis, but an experience with the system during some early check runs provided some indirect evidence to support this hypothesis. The atmospheric venting assembly downstream of the mass flow plug was clogged by $25 \%$ area in one digital vent valve and the inlet mass flow was reduced by 20 to $22 \%$. This suggests that this vent area is the bottleneck in the inlet mass flow system design. If this vent valve were the limiting flow restriction, then to increase the operational inlet flow rate range of the existing inlet model system, an increase to this atmospheric vent valve area would be useful. However, for the current investigation the inlet mass flow ratio was limited to 0.55 and below.

\section{Instrumentation and control jet system}

The inlet instrumentation included surface pressure ports along the top, bottom and sidewalls of the diffuser. A total pressure rake with 40-probes was positioned at the AIP to measure pressure recovery and distortion. A portion of this rake is seen in figure $4 \mathrm{a}$. The total pressure rake was designed to conform to the SAE standard. ${ }^{35}$ Each rake arm is separated from the next by $45^{\circ}$. The frontal area for all eight-rake arms produced almost $15 \%$ blockage of the AIP area. All of these pressure probes were connected to the tunnel electronic scanning pressure measurement systems.

The air supply system for the AFC jets tapped into the tunnel 350-psig air system and was connected to a pressure regulator that controlled the supply pressure to the air supply panel, which is pictured in figure 6a. The supply flow passes through this regulator and an air filter until it reaches a thermal measurement mass-flow meter. The mass flow meter had a flow rate measurement range up to $0.08 \mathrm{lbm} / \mathrm{s}$. After passing through the mass flow meter, the control jet mass flow is split at a piping T-junction to supply two separate actuator manifolds. A pressure transducer located on one of the pressure manifolds provided an indication of the supply pressure to the high mass flow actuators, which are shown in figure $6 \mathrm{~b}$. These actuators are compressed natural gas fuel injectors that acted as solenoid valves, which could be operated in either a steady or pulsed mode. In the steady mode of operation, the 
actuators are just held open and act as switches for turning on and off certain regions of control jets. In the pulsed mode, the actuators have a frequency range of 20 to $200 \mathrm{~Hz}$ and a duty cycle (\% of time during a pulsing cycle when the valve is open) range of 20 to $80 \%$. The actuators were located outside the tunnel early in the test setup design because the original plan included cryogenic test conditions. As the test setup evolved, it became clear that a dry high-pressure gas supply would not be available and all cryogenic test conditions were dropped from the test matrix. The location of the actuators outside the tunnel created excessively long control jet supply tubing lengths that contained many joints/restrictions. The control jet supply tubing connected to each actuator on one end (see figure $6 \mathrm{~b}$ ) and then was split to supply the control jets for the desired distribution (see figure 7a) to finally pass through the tunnel pressure shell (see figure $7 \mathrm{~b}$ ) to connect to the control jet orifices in the inlet model. The different control jet distribution configurations enabled a tubing hook up of one actuator to two, four or six control jet tubes. The number of control jets per actuator was always even because of the symmetry constraint imposed across the centerline of the diffuser. If one actuator is distributed to two control jets, then the jet velocity is generally higher than if connected to four or more control jets given the same number of actuators supplying the flow.

The tubing length and restrictions resulting from this test setup attenuated the actuator pulsed jet response such that the signal was essentially steady even when the actuators were operated in a pulsing mode. So, all the active flow control testing was performed with steady control jets and the actuators were only used in the steady mode as switches for regions of flow control jets. The omission of the pulsed control jets testing left a potential research question of whether pulsing jets could leverage some flow feature inside the diffuser to achieve a given level of distortion reduction with reduced control jet mass-flow rate requirements.

One of the control jet tubes was instrumented with a pressure transducer within six inches of the jet orifice. Similarly, another jet tube was instrumented with a thermocouple within about six inches of the jet orifice. The measurements were added to provide some guidance for setting jet boundary conditions for CFD simulations. Further analysis of this data is still needed, but some general values for these measurements are included for reference. The jet temperature was consistent for the entire test over all the test conditions covered. The jet temperature generally stayed about $70^{\circ} \mathrm{F}$, which was close to the measured air supply temperature. The jet pressure varied as the jet mass flow ratio increased. The maximum pressure level observed was about 75 psia at the maximum control jet mass flow rates. This jet pressure expanded into a diffuser with measured pressure levels around 25 psia for the tunnel total pressure test condition of 30 psia. So, the maximum jet pressure ratio tested was approximately three.

An estimate of the uncertainty levels for key parameters is provided in table 3. These uncertainty estimates were developed using procedures outlined in reference 36.

\section{Boundary Layer Assessment}

Before studying the effects of BLI, assessment of the degree of BLI for the test setup was needed. The inlet model was scaled in the design process to achieve $30 \%$ BLI, which means that the onset flow boundary layer height would be $30 \%$ of the inlet throat height. The inlet influences this oncoming boundary layer height, so it is not obvious where one should measure a varying quantity around the inlet model. The method used in the investigation in reference 31 was to measure the boundary layer at the same tunnel station as the inlet lip highlight, but offset to one side of the inlet. This method was chosen so that relevant comparisons could be made with earlier research on the baseline inlet model. A different boundary layer rake from that used in the research of reference 31 was chosen to get more detailed measurements within the boundary layer. The boundary layer rake used in the current study is shown in figure 4. Some of the measurements obtained during this test are shown in figure 8 along with an inset view of the installed inlet model and boundary layer rake for reference. The analysis of this data suggested that the boundary layer height was insensitive to Mach number changes and inlet mass-flow adjustments (XPLUG variation). The boundary layer height was approximately 0.6 inches. In the detailed analysis of the boundary layer properties, the measured data produced five to six data points in the fitted log region of the law of the wall plot. The law of the wall model with East's buffer region was used to integrate the boundary layer profile to the wall. The integration of the compressible boundary layer profile for the $\mathrm{M}=0.84$ data gave a shape factor $\left(\mathrm{H}=\delta^{*} / \theta\right)$ of about 1.5 , which is consistent with what is expected for a compressible, turbulent, flat-plate boundary layer. Also, for reference the same boundary layer rake had been tested earlier in the empty test section at the location noted in the inset picture in figure 8. The empty test section rake measurement is in a different location, but provides another perspective to assess the boundary layer height on this tunnel wall. The inlet model test boundary layer profile measurements are similar to the empty test section boundary layer profile. Taking the ratio of boundary layer height of about 0.6 inches to the inlet model throat height of about 1.7 inches gives a value for the degree of BLI at about $35 \%$. 
The boundary layer rake was removed after the initial measurements were made for the test conditions covered in this test. The rake was removed because of the blockage effect that produced an asymmetric pressure distribution at the AIP. The rake blockage was significant enough to produce supersonic flow (calculated using local wall pressures) and the potential of shock interactions with the inlet flow field became a concern. Figure 9 shows a comparison of the AIP rake pressure contours with and without the boundary layer rake installed for various inlet mass flow conditions. The boundary layer rake was on the side of the inlet that corresponded to the left side of the pressure contour plots shown in this figure. These pressure contours became more symmetric with the boundary layer rake removed. The rest of the test was performed without additional boundary layer measurements. Note that these asymmetries in the AIP pressure measurements may be a realistic installed situation for a BWB configuration if the neighboring inlet produces similar levels of interference effects.

\section{E. Adaptive Tunnel Walls Setup}

The inlet model scale $(2.5 \%)$ was chosen to give a certain degree of BLI. This design approach produced a model that is large relative to the tunnel test section size, especially at transonic test conditions. The adaptive tunnel walls, which lay on either side of the inlet model, were used to try and reduce some of the expected wall interference. The adaptive wall control system was developed to automatically streamline these walls around airfoil shapes that span the test section. The inlet model did not span the test section and was not suited for this type of automatic streamlining setup. So, the adaptive wall positions were manually set during the early check runs for the test and the resulting area distribution was iterated until an acceptable Mach number distribution was obtained throughout the test section length. The final adaptive wall positions that were used throughout the test are shown in figure 10. The delta wall positions plotted in the bottom of this figure show each wall displacement from a parallel wall position. Notice that the wall shapes ramp into and out of a bulbous area coincident to the inlet location. Ideally, the first four wall positions would be zero and then very rapidly the fifth position would transition to the bulbous region. However, the limited number of wall jack locations in this upstream region made it necessary to open up the walls earlier than needed to keep from stretching the wall material between neighboring jack locations while displacing the walls to the extent needed. The result of the wall ramping was a varying local Mach number after entering the test section.

The method chosen to finally set the wall shape was to set a shape to produce a somewhat flattened local Mach number distribution and then adjust the test section entrance Mach number to 0.88 to obtain $\mathrm{M}=0.85$ flow ahead of the inlet. The local Mach distributions used to determine whether or not the wall shaping was sufficient were calculated from the wall pressure locations shown as filled circles in figure 11. Figure 12 presents a typical local Mach number distribution with the final wall shape used throughout the test. The flow enters the test section at $\mathrm{M}=0.88$ and begins to slow down to about $\mathrm{M}=0.85$ before reaching the inlet model location. After reaching the inlet, the flow accelerates slightly before dropping to a new Mach number level after the inlet. This Mach level drop after the inlet is caused by the tunnel mass flow removal by the inlet that cannot be replaced and appears to the flow as an effective cross-sectional area increase. This type of local Mach distribution was the best one obtained given the resources available to address this issue and was considered acceptable to continue with the flow control testing.

\section{F. Flow Control Devices}

Two types of flow control devices were used to manipulate the flow inside the inlet diffuser. The two devices were air jets and vortex generators. The general layout of the two types of flow control devices is shown in figure 13. The view is of the left half of the inlet diffuser as seen from a downstream, three-quarters-view perspective. In the figure on the left, the available air control jet locations are generally shown. The CFD simulations discussed in reference 32 guided the selection of these jet locations. There are 176 control jet orifice locations distributed along 11 different axial stations. Each jet orifice diameter is 0.040 inches. Each jet was skewed $90^{\circ}$ to the oncoming flow and pitched up $30^{\circ}$ from the local surface tangent. This produced jets that generally produced a sideways deflection of the oncoming flow. This approach was taken to counter the secondary inlet flow that is produced by the higher pressure at the top of the diffuser than at the bottom, which tends to cause low momentum flow to pool in the bottom of the diffuser. So, the jets were oriented to primarily impart momentum to produce a side force on the local flow and secondly to create vorticity.

The vortex generator (VG) configuration tested is shown on the right side of figure 13. The layout of these VGs were obtained from a design-of-experiments CFD analysis at a given inlet flow condition. Details describing this analysis can be found in reference 37. The VG design placed groups of six in four different regions toward the front of the diffuser. Two of these groups are shown in figure 13, one on the left bottom of inlet and the other on the left side of the inlet. The VG groups on the bottom of the inlet had a design height of 0.181 inches $\left(h / D_{2}=0.074\right)$ and 
were angled $12.9^{\circ}$ to the oncoming free stream flow. The VG groups on the sides of the inlet had a design height of 0.163 inches $\left(\mathrm{h} / \mathrm{D}_{2}=0.065\right)$ and were angled $11.9^{\circ}$ to the oncoming free stream flow.

\section{Discussion of Results}

\section{A. Effects of control jets on inlet flow distortion and pressure recovery}

The general character of the control jets on the circumferential distortion $\left(\mathrm{DPCP}_{\text {avg }}\right)$ as calculated using the method described in the SAE standard ${ }^{35}$ is shown in figure 14. The control jet distribution used for this active flow control case is shown in the inset picture in the upper right-hand side of the figure for reference. The distortion is plotted versus the total control jets mass flow rate normalized by the inlet mass flow rate for this test condition. The character of these distortion curves is an initial distortion plateau region that occurs from 0 to $0.75 \%$ control jet mass flow ratio. Above a control jet mass flow ratio of $0.75 \%$, the distortion begins to decrease until reaching a minimum distortion level at a jet mass flow ratio of $2.5 \%$. The different distortion curves for each tunnel total pressure (or $\mathrm{Re}, \mathrm{D}$ ) were run in an attempt to find the highest Reynolds number level (i.e., total pressure level) that would allow the fixed maximum control jets mass flow rate to span the highest to the lowest distortion levels. For this test, the total pressure level needed to be lowered to 30 psia to enable the control jets to cover this range, which essentially increased the available range of the ratio of jet pressure to the internal diffuser pressure (i.e., jet pressure ratio). The doubling of the tunnel total pressure also doubled the Reynolds number. Over the Reynolds number range covered there did not appear to be any significant influence of the Reynolds number on the distortion levels.

Physical insights into the character of these distortion curves are gained by examining the total pressure contours from measurements at the AIP. These contours are shown for four different distortion levels along these distortion curves. Starting at the far left, the pressure contour shown is typical for the baseline (no flow control) inlet flow field. The upper half of the AIP has high pressure levels, which represents undistorted inlet flow. The bottom half of the AIP has low pressure regions characteristic of low momentum boundary layer flow that pools at the bottom of the inlet. This flow collects at the bottom of the inlet because of the secondary flow generated by the pressure difference between the upper (high pressure) and lower (low pressure) surfaces of the forward part of the diffuser. The plateau region in the distortion curve results from all the jet momentum going towards balancing the effect of this secondary flow. So, up to a jet mass flow ratio of $0.75 \%$ there has been no real progress yet in dealing with the BLI problem. The next pressure contour at the jet mass flow ratio of $1.0 \%$, was selected to demonstrate why the distortion level is starting to decrease. In this contour plot, the low pressure region at the bottom of the diffuser has begun to be spread around the circumference of the AIP. At this point, the control jets have balanced the secondary flow forces and have only just begun to deal with the BLI problem itself. The next two pressure contours show the effects of the increased jet momentum to continue to spread the boundary layer around the circumference of the diffuser, which continues to decrease the circumferential distortion level. The last pressure contour begins to show signs of the boundary layer flow collecting on the sides of the AIP at the minimum distortion level attained. This pooling of the low momentum flow on the sides of diffuser suggests that the jet distribution is no longer adequate to effectively deal with the BLI problem. The bottom jets for this configuration have cleared too much of this flow from the bottom of the diffuser and there are also not enough side jets to help clear some of this flow from the sides toward the top of the AIP. So, the inefficiency of the jet distribution at this distortion level explains why the distortion level has hit a minimum here.

The pressure recovery trends that correspond to the distortion curves discussed above are presented in figure 15 . In general, the pressure recovery decreases approximately 0.01 as the distortion level decreases. This results because the average total pressure at the AIP decreases as more of the low momentum flow is distributed among more of the AIP pressure probes. Basically, the decrease in the average total pressure may only be a measurement resolution issue. The low momentum flow that occurs between the AIP rakes on the bottom of the diffuser is spread around the circumference of the AIP so that it becomes measurable by more of the pressure probes. So, the pressure recovery is probably fairly constant over the range of jet mass flow ratios covered and the pressure recovery level is more likely to be near the lowest level shown.

The pressure recovery trends did indicate a small effect of increasing Reynolds number. In the regions of the pressure recovery curves where the data overlap, there are signs of increasing pressure recovery as the Reynolds number increased. This Reynolds number trend is consistent with that observed in the baseline inlet Reynolds number investigation described in reference 31. The pressure recovery is generally expected to increase as flow deficits are reduced. Increasing the Reynolds number decreases the boundary layer thicknesses, which would produce this effect. 


\section{B. Effects of jet velocity and distribution on inlet flow distortion and pressure recovery}

The impact of different control jet velocities and jet distributions on the effectiveness to reduce the circumferential inlet distortion is presented in figure 16. The distortion trends presented were selected to represent some of the configurations tested that most effectively reduced the circumferential distortion. An inset picture of the jet distribution pattern is included for each of the distortion curves to give a sense of how the jet distribution correlates to the distortion reduction effectiveness. The jet patterns with the lowest number of jets tended to overcome the secondary flow and started reducing the distortion at lower control jet mass flow ratios than those with a higher number of control jets. This behavior appears to be related to the higher jet velocities producing initially more effective distortion reduction configurations. However, comparing the two distortion curves for the two configurations with 16 jets shows the importance of the jet distribution pattern. One of these two configurations shifted the jet distribution from being concentrated near the diffuser bottom centerline to spreading the jets more toward the sides of the diffuser. In this comparison, the jet velocities would be similar such that the jet distribution is the primary driver in each pattern's distortion reduction effectiveness. The configurations which tended to show the largest reductions in the inlet distortion tended to have more jet orifices distributed along the sides of the diffuser. Referring back to the pressure contour for the minimum distortion level shown in figure 14, increasing the number of jets on the sides of the diffuser to keep the spreading boundary layer from collecting on the sides seems to be a necessary approach in dealing with the BLI inlet distortion problem. So, the most effective distortion reduction configurations are those that effectively balance both the jet velocity (number of jets) and the distribution of those jets inside the inlet diffuser. Higher jet velocities for jet orifices evenly distributed between the forward bottom and sides of the diffuser are potentially the best jet patterns to consider for this type of active flow control application.

The pressure recovery trends for the different control jet patterns with varying control jet mass flow ratios are shown in figure 17. The general trend of pressure recovery loss with increasing control jet mass flow rate is similar to that discussed in figure 15 . However, the 30 jet configuration did show a little higher pressure recovery for jet mass flow ratios above $1.5 \%$.

\section{Effects of circumferential distortion reduction on radial distortion}

Redistributing the ingested boundary layer around the circumference of the AIP causes the reduction of the circumferential distortion in the inlet. This redistribution of the low momentum flow reduces the gradients circumferentially but increases them in the radial direction. A radial distortion pattern representative of all the flow control data acquired during the current test is shown in figure 18. Each curve corresponds to a different circumferential inlet distortion level. The magnitude of the radial distortion consistently increased as the circumferential distortion levels decreased. In the flow control approach taken to deal with this type of BLI problem, the boundary layer is not removed. Instead, the boundary layer is shifted from one gradient pattern to another. So, the effectiveness of this approach will need to be evaluated by which distortion constraint is the most important to control during BLI inlet operations.

\section{Effect of VGs alone control and Hybrid VG/air jet control on distortion reduction and pressure recovery}

The variation of the inlet circumferential distortion with changing inlet mass flow ratio for both the baseline (no flow control) and VG configurations is shown in figure 19. Both of these configurations are pictured in figure 20 and figure 21 shows the VG layout more clearly. Details about the design of the VGs are included in reference 37. In figure 19, the distortion trends with the inlet mass flow ratio show that the VG configuration was effective in reducing the inlet distortion level for almost the entire range of inlet mass flow settings. The baseline configuration distortion levels increased almost linearly with increasing inlet mass flow rate. The VG configuration distortion response was somewhat nonlinear over the range of inlet mass flow rates covered in this test. The VG configuration was essentially a point design at an inlet mass flow ratio of 0.59 . The AIP pressure contour in the lower right-hand portion of the figure demonstrates how effective the VGs are at evenly redistributing the boundary layer flow around the circumference of the diffuser exit, especially when compared to the baseline AIP pressure contour plot just above it.

For the VG configuration distortion, reducing the inlet mass flow rate below the design point caused the distortion to increase because of the reduced effectiveness of the VGs. Preliminary CFD simulations of the VG configuration over this range of inlet mass flow rates have provided some indications for why the VG effectiveness decreases. The simulations tend to show increased inlet flow spillage as the inlet mass flow rate is decreased, which is closely coupled with the increase in the size of two juncture vortices that reduces the velocity of the flow approaching the VGs.

The variation of the inlet pressure recovery with changing inlet mass flow rates for both the baseline and VG configurations is shown in figure 20. The baseline pressure recovery increases as the inlet mass flow rate increases 
while that for the VG configurations does not change. Notice that the pressure recovery reductions between the baseline and VG configurations are consistent with the reductions observed for the control jet configurations.

Finally, the VG configuration had an increased distortion level for reduced inlet mass flow rates (see figure 19) and a configuration was tested that added four control jets to work a short distance downstream of the VGs. An inset picture of the hybrid VG/jet control configuration is presented on the right side of figure 21. The reason for combining these two types of flow control devices was to balance the strength and weaknesses of each device. The control jets require a significant amount of mass flow rate to effectively reduce the inlet distortion, but do not depend on the velocity of the onset flow to create a flow deflection force. The VGs do not require any mass flow rate to effectively reduce the inlet distortion, but do depend on the onset flow velocity to create a flow deflection force. Together, the VGs can reduce most of the inlet distortion and the control jets can be used at a significantly reduced control jet mass flow rate to make sure the inlet distortion stays low as the inlet mass flow rate varies. To demonstrate this concept, the hybrid configuration was tested at inlet mass flow rates where the distortion level was elevated for the VG configuration and added four control jets to reduce the inlet distortion. The significantly reduced control jet mass flow rate to accomplish this is shown in the data presented in figure 21.

\section{Conclusion}

The goal of this research was to determine whether active flow control could be used to control the distortion levels for an S-inlet diffuser with significant BLI (35\%). A systematic approach was taken to develop an actuator system with control jets configured to manage the inlet flow field. The active flow control system had to manage two basic flow mechanisms inside the inlet, the secondary flow and the significant BLI. There is a large body of research that deals with active flow control handling the secondary flow at transonic Mach conditions. This study is unique because it is the first known research to deal with both of these mechanisms at transonic Mach numbers for a range of inlet operating conditions. The investigation included different control jet configurations and one VG configuration. During the investigation, the following was learned:

- The high Mach number BLI inlet testing provided a small-scale demonstration of the ability of an active flow control system to reduce the inlet flow distortion (circumferential). Although the system developed is not optimal, insights were gained that can guide future research in this area.

- For $\mathrm{M}=0.85$, the application of active flow control steady jets operating at $1.5 \%$ of inlet mass flow for one jet distribution reduced the distortion from a $\mathrm{DPCP}_{\text {avg }}$ value of 0.055 for the baseline to 0.025 for the active flow control case. This configuration did not provide the lowest distortion level, but did provide the most effective one by significantly reducing the distortion with the least amount of control jet mass flow rate. Increasing the control jets to $2.5 \%$ of the inlet mass flow for another jet distribution continued the distortion reduction to about 0.015 . This configuration was considered the most effective at generating the lowest distortion levels.

- Balancing both the control jet velocity as well as the jet distribution is important in reducing inlet circumferential distortion. The jet velocity needs to be as high as possible to reduce the distortion with as few jets as possible to keep the jet mass flow rate relatively low. However, the number of jets needs to be sufficient to distribute them in the forward middle region of the diffuser and down the diffuser sides to keep the cleared out boundary layer from collecting on the sides of the diffuser.

- The measured pressure recovery decreases with significant BLI inlet flow control. This occurs because the ingested boundary layer is not removed with this approach so that the circumferentially redistributed boundary layer affects more of the AIP probes and reduces the average total pressure at the diffuser exit. This flow control character occurs for both the control jet and the VG configurations.

- The point design VG configuration effectively reduced the circumferential distortion as compared to the baseline configuration. The distortion level increased for the VG configuration when the inlet mass flow rate was reduced.

- The hybrid system that combined both VGs and control jets worked to keep the inlet distortion level low across the range of inlet mass flow rate settings.

\section{Acknowledgments}

This research was supported by the NASA Ultra Efficient Engine Technology (UEET) Highly Integrated Inlet (HII) project. Special thanks goes to Mr. Bobby Berrier for his guidance in the area of testing flush-mounted inlets. The authors would also like to acknowledge Mr. Tony Washburn and Ms. Judi Hannon for the significant 
contributions to the data acquisition hardware and software. Finally, special thanks to the entire NASA 0.3-meter TCT staff who helped make the experiment a successful one, especially for Ms. Stacy Sigmon.

\section{References}

\footnotetext{
${ }^{1}$ Johnson, T., “Aviation's Environmental Impact on the Global Atmosphere," Proceedings of Aviation and the Environment Their Future in an Integrated Transport Policy, RAE, London, 1999, pp. 13.1-13.5.

${ }^{2}$ Green, J. E., "Air Travel- Greener by Design, Mitigating the environmental impact of aviation: Opportunities and priorities," The Aeronautical Journal of the Royal Aeronautical Society, v 109, no 1099, September 2005, pp. 361-416.

${ }^{3}$ Brown, A. S., "HSR Work Propels UEET Program (High Speed Research in Ultra-efficient Engine Technology in Aircraft Industry)," Aerospace America, Vol. 37, No. 5, May 1999, pp. 48-50.

${ }^{4}$ Callaghan, J. T., and Liebeck, R. H., "Some Thoughts on the Design of Subsonic Transport Aircraft for the $21^{\text {st }}$ Century," Cockpit, Dec. 1990, pp. 5-13.

${ }^{5}$ Liebeck, R. H., Page, M. A., Rawdon, B. K., "Evolution of the Revolutionary Blended-Wing-Body," Transportation Beyond 2000: Technologies Needed for Engineering Design," February, 1996, pp. 431-459.

${ }^{6}$ Liebeck, R. H.,'Design of the Blended-Wing-Body Subsonic Transport," AIAA 2002-002.

${ }^{7}$ Daggett, D. L., "Ultra Efficient Engine Technology Systems Integration and Environmental Assessment," NASA CR-2002211754, July 2002.

${ }^{8}$ Anabtawi, A. J., Blackwelder, R. F., Lissaman, P. B. S., Liebeck, R. H., “An Experimental Investigation of Boundary Layer Ingestion in a Diffusing S-Duct With and Without Passive Flow Control," AIAA 99-0739.

${ }^{9}$ Anderson, B. H., and Gibb, J., "Vortex Generator Installation Studies on Steady State and Dynamic Distortion," AIAA 963279, July, 1996.

${ }^{10}$ Lin, J. C., "Review of Research on Low-Profile Vortex Generators to Control Boundary-Layer Separation," Progress in Aerospace Sciences, Vol 38, p 389-420, 2002.

${ }^{11}$ Anderson, B. H., Baust, H. D., and Agrell, J., "Management of Total Pressure Recovery, Distortion and High Cycle Fatigue in Compact Air Vehicle Inlets," NASA TM-2002-212000, December, 2002.

${ }^{12}$ Anderson, B. H., Miller, D. N., Yagle, P. J., and Truax, P. P., "A Study on MEMS Flow Control For the Management of Engine Face Distortion in Compact Inlet Systems," Proceedings of the $3^{\text {rd }}$ ASME/JSME Joint Fluids Engineering Conference, July, 1999.

${ }^{13}$ Hamstra, J. W., Miller, D. N., Truax, P. P., Anderson, B. H., and Wendt, B. J., “Active Inlet Flow Control Technology Demonstration," The Aeronautical Journal of the Royal Aeronautical Society, October, 2000.

${ }^{14}$ Seddon, J., and Goldsmith, E. L., Intake Aerodynamics, Second Edition, AIAA, Reston, Virginia, 1999, p. 169, $278-280$.

${ }^{15}$ Gorton, S. A., Owens, L. R., Jenkins, L. N., Allan, B. G., and Schuster, E. P., “Active Flow Control on a Boundary-LayerIngesting Inlet, " AIAA-2004-1203.

${ }^{16}$ Lord, W. K., MacMartin, D. G., and Tillman, T. G., "Flow Control Opportunities in Gas Turbine Engines," AIAA 20002234.

${ }^{17}$ Vakili, A. D., Wu, J. M., Liver, P., and Bhat, M. K., "Flow Control in a Diffusing S-Duct," AIAA 85-0524.

${ }^{18}$ Smith, B. L., and Glezer, A., "The Formation and Evolution of Synthetic Jets," Physics of Fluids, Vol. 10, No. $9,1998$.

${ }^{19}$ Barberopoulos, A. A., and Garry, K. P., "The Effect of Skewing on the Vorticity Produced by an Airjet Vortex Generator," The Aeronautical Journal of the Royal Aeronautical Society, v 102, no 1013, March 1998, pp. 171-179.

${ }^{20}$ Tilmann, C. P., Langan, K. J., Betterton, J. G., and Wilson, M. J., "Characterization of Pulsed Vortex Generator Jets for Active Flow Control," Presented at the RTO AVT Symposium on Active Control Technology for Enhanced Performance Operation Capabilities of Military Aircraft, Land Vehicles and Sea Vehicles, Germany, May, 2000.

${ }^{21}$ Peake, D. J., Henry, F. S., and Pearcey, H. H., "Viscous Flow Control with Air-Jet Vortex Generators," AIAA 99-3175, June, 1999.

${ }^{22}$ Crook, A., and Wood, N. J., "Measurements and Visualizations of Synthetic Jets," AIAA 2001-0145.

${ }^{23}$ Wygnanski, I., "Some New Observations Affecting the Control of Separation by Periodic Excitation," AIAA 2000-2314, June, 2000.

${ }^{24}$ Washburn, A. E., Gorton, S. A., and Anders, S. G., "Snapshot of Active Flow Control Research at NASA Langley," AIAA 2002-3155.

${ }^{25}$ Schaeffler, N. W., Hepner, T. E., Jones, G. S., and Kegerise, M. A., "Overview of Active Flow Control Actuator Development at NASA Langley Research Center," AIAA 2002-3159.

${ }^{26}$ Sellers, W. L., III, Jones, G. S., and Moore, M. D., "Flow Control Research at NASA Langley in Support of High-Lift Augmentation," AIAA 2002-606.

${ }^{27}$ Rao, N. M., Feng, J., Burdisso, R. A., and Ng, W. F., "Experimental Demonstration of Active Flow Control to Reduce Unsteady Stator-Rotor Interaction," AIAA Journal, v 39, no 3, March 2001, p 458-464.

${ }^{28}$ Jenkins, L. N., Gorton, S. A., and Anders, S. G., "Flow Control Device Evaluation for an Internal Flow with an Adverse Pressure Gradient," AIAA 2002-0266.

${ }^{29}$ Berrier, B. L., "Evaluation of Flush-Mounted, S-Duct Inlets with Large Amounts of Boundary Layer Ingestion," RTO Vehicle Propulsion Integration Symposium, Warsaw, Poland, October, 2003.
} 
${ }^{30}$ Berrier, B. L., and Allan, B. G., "Experimental and Computational Evaluation of Flush-Mounted, S-Duct Inlets (Invited), ” AIAA-2004-0764.

${ }^{31}$ Berrier, B. L., Carter, M. B., and Allan, B. G., "High Reynolds Number Investigation of a Flush-Mounted, S-Duct Inlet with Large Amounts of Boundary Layer Ingestion," NASA TP-2005-213766, September 2005.

${ }^{32}$ Allan, B. G., Owens, L. R., and Berrier, B. L., "Numerical Modeling of Active Flow Control in a Boundary Layer Ingesting Offset Inlet, " AIAA-2004-2318.

${ }^{33}$ Mineck, R. E., and Hill, A. S., "Calibration of the 13- by 13-Inch Adaptive Wall Test Section for the Langley 0.3-Meter Transonic Cryogenic Tunnel, ”NASA TP-3049, December 1990.

${ }^{34}$ Rallo, R. A., Dress, D. A., and Siegle, H. J. A., "Operating Envelope Charts for the Langley 0.3-Meter Transonic Cryogenic Wind Tunnel, "NASA TM-89008, August 1986.

${ }^{35}$ Gas Turbine Engine Inlet Flow Distortion Guidelines. Aerospace Recommended Practice 1420B, SAE International, 2001.

${ }^{36}$ Instruments and Apparatus. "Part I - Measurement Uncertainty," ANSI/ASME PTC 19.1-1985, American National Standards Inst., 1985.

${ }^{37}$ Allan, B. G., Owens, L. R., and Lin, J. C., "Optimal Design of Passive Flow Control for a Boundary-Layer-Ingesting Offset Inlet Using Design-of-Experiments, ” AIAA-2006-1049. 


\section{Tables}

Table 1. Inlet Characteristics

\begin{tabular}{|r|c|}
\hline Average wall angle $(\phi)[\mathrm{deg}]$ & 18.3 \\
\hline Inlet length $(\mathrm{L})[\mathrm{in}]$. & 7.696 \\
\hline Inlet offset height $(\Delta \mathrm{H})[\mathrm{in}]$. & 2.543 \\
\hline Exit diameter $\left(\mathrm{D}_{2}\right)[\mathrm{in}]$. & 2.448 \\
\hline Inlet throat area $\left(\mathrm{A}_{\mathrm{i}}\right)$ [sq. in.] & 4.400 \\
\hline Inlet capture (highlight) area $\left(\mathrm{A}_{\mathrm{C}}\right)$ [sq. in.] & 5.760 \\
\hline Diffuser exit area $\left(\mathrm{A}_{2}\right)[\mathrm{sq}$. in.] & 4.704 \\
\hline Inlet throat height $\left(\mathrm{H}_{\mathrm{i}}\right)[\mathrm{in}]$. & 1.703 \\
\hline Inlet throat width $\left(\mathrm{W}_{\mathrm{i}}\right)[\mathrm{in}]$. & 3.249 \\
\hline Inlet lip length $(\mathrm{a})[\mathrm{in}]$. & 0.479 \\
\hline Inlet lip height $(\mathrm{b})[\mathrm{in}]$. & 0.240 \\
\hline Cowl forebody length $(\mathrm{X})[\mathrm{in}]$. & 0.713 \\
\hline Cowl maximum height $\left(\mathrm{H}_{\text {max }}\right)[\mathrm{in}]$. & 2.185 \\
\hline Cowl aftbody length $\left(\mathrm{X}_{\text {aft }}\right)[\mathrm{in}]$. & 14.610 \\
\hline Cowl aftbody boattail angle $[\mathrm{deg}]$ & 11.0 \\
\hline Design throat Mach number & 0.70 \\
\hline
\end{tabular}

Table 3. Uncertainty Estimates

\begin{tabular}{|c|c|}
\hline Parameter & Uncertainty \\
\hline $\mathrm{M}$ & \pm 0.003 \\
\hline $\mathrm{P}_{\mathrm{t}}$ & $\pm 0.1 \mathrm{psia}$ \\
\hline $\mathrm{T}_{\mathrm{t}}$ & $\pm 0.2^{\circ} \mathrm{F}$ \\
\hline $\mathrm{U}_{\mathrm{e}}$ & \pm 0.01 \\
\hline $\mathrm{P}_{\mathrm{t}, 2}$ & $\pm 0.06 \mathrm{psia}$ \\
\hline Spatial distances & \pm 0.005 inches \\
\hline Distortion & \pm 0.001 \\
\hline Pressure Recovery & \pm 0.004 \\
\hline Inlet mass flow & $\pm 0.02 \mathrm{lbm} / \mathrm{s}$ \\
\hline Control Jet mass flow & $\pm 0.002 \mathrm{lbm} / \mathrm{s}$ or $\pm 0.1 \%$ of \\
& $\begin{array}{c}\text { Inlet Mass Flow Rate as } \\
\text { plotted (referenced to an } \\
\text { inlet mass flow rate of } 2 \\
\end{array}$ \\
& lbm/s) \\
\hline $\mathrm{A}_{\mathrm{o}} / \mathrm{A}_{\mathrm{C}}$ & \pm 0.006 \\
\hline
\end{tabular}

Table 2. Predicted BWB Full/ Model-scale corrected inlet airflow values

\begin{tabular}{|c|c|c|c|c|c|c|}
\hline Altitude, $\mathbf{f t}$ & Mach & Condition & $\begin{array}{c}\text { Net Thrust, } \\
\text { lbf }\end{array}$ & $\begin{array}{c}\text { Full-scale } \\
\text { Corrected } \\
\text { Airflow, } \\
\text { lbm/s }\end{array}$ & $\begin{array}{c}2.5 \% \text {-scale } \\
\text { Corrected } \\
\text { Airflow, } \\
\text { lbm/s }\end{array}$ & $\mathbf{A}_{\mathbf{O}} / \mathbf{A}_{\mathbf{C}}$ \\
\hline 36089 & 0.85 & Max Climb & 14555 & 2081 & 1.30 & 0.65 \\
\hline & & Max Cruise & 13976 & 2062 & 1.29 & 0.64 \\
\hline & & Part Power & 13277 & 2038 & 1.27 & 0.63 \\
\hline & & 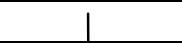 & 12590 & 2012 & 1.26 & 0.62 \\
\hline & & & 11180 & 1950 & 1.22 & 0.60 \\
\hline & & & 9784 & 1882 & 1.18 & 0.58 \\
\hline & & & 8384 & 1811 & 1.13 & 0.56 \\
\hline & & & 6989 & 1737 & 1.09 & 0.54 \\
\hline & & & 5591 & 1659 & 1.04 & 0.51 \\
\hline & & & 4193 & 1559 & 0.97 & 0.48 \\
\hline$\nabla$ & $\nabla$ & $\checkmark$ & 2793 & 1468 & 0.92 & 0.46 \\
\hline
\end{tabular}




\section{Figures}

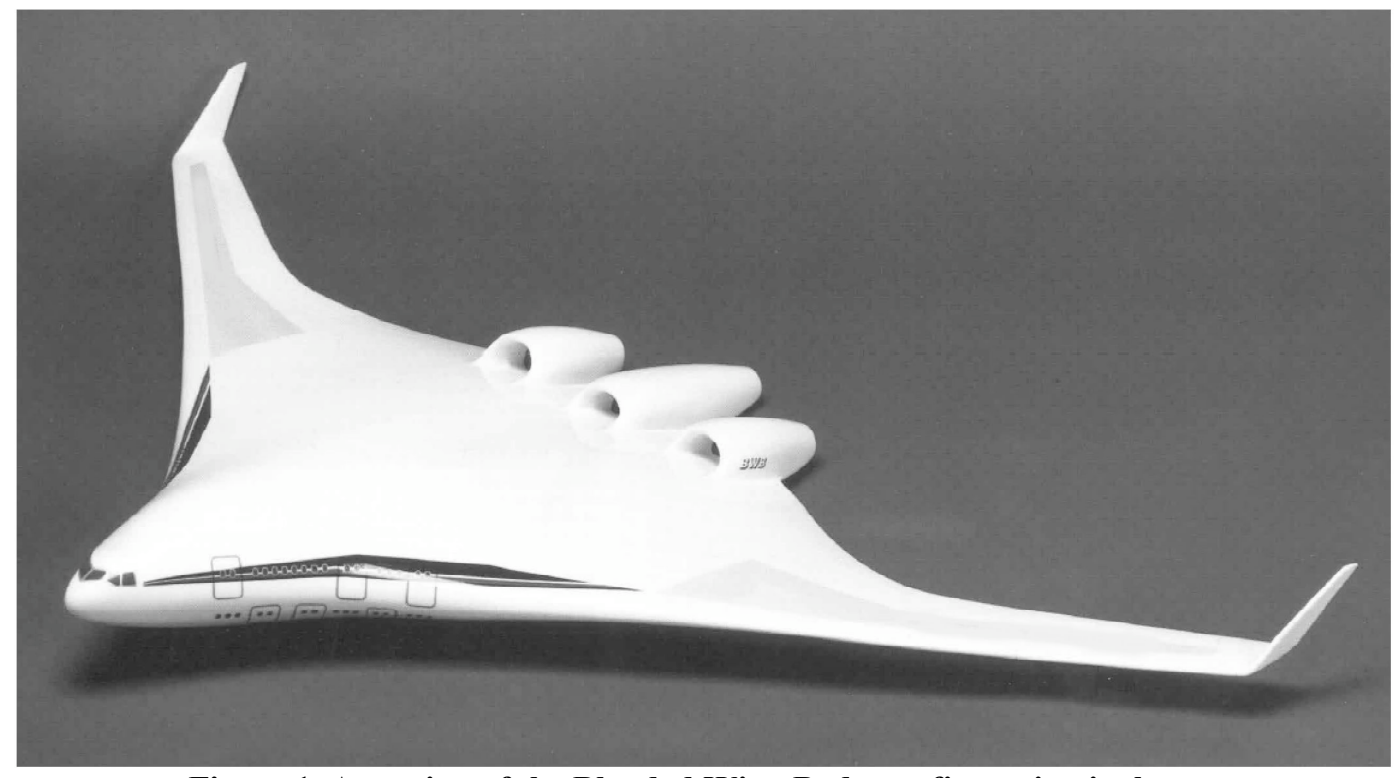

Figure 1. A version of the Blended Wing Body configuration is shown.

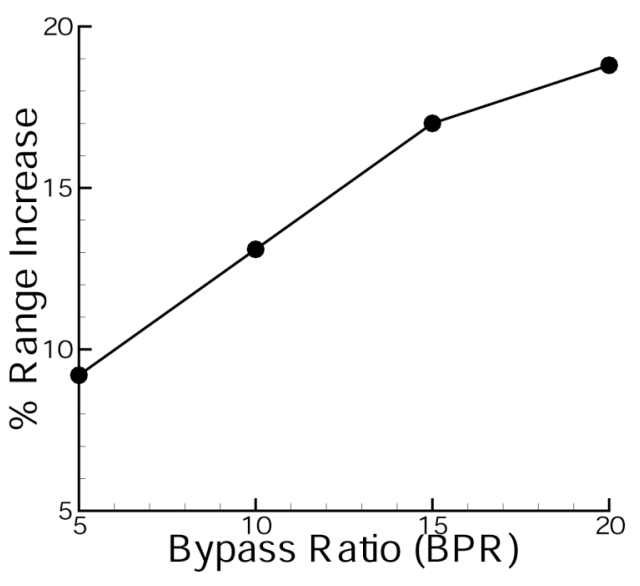

Figure 2. The Effect of BLI on range increase as a function of Bypass Ratio (BPR). 


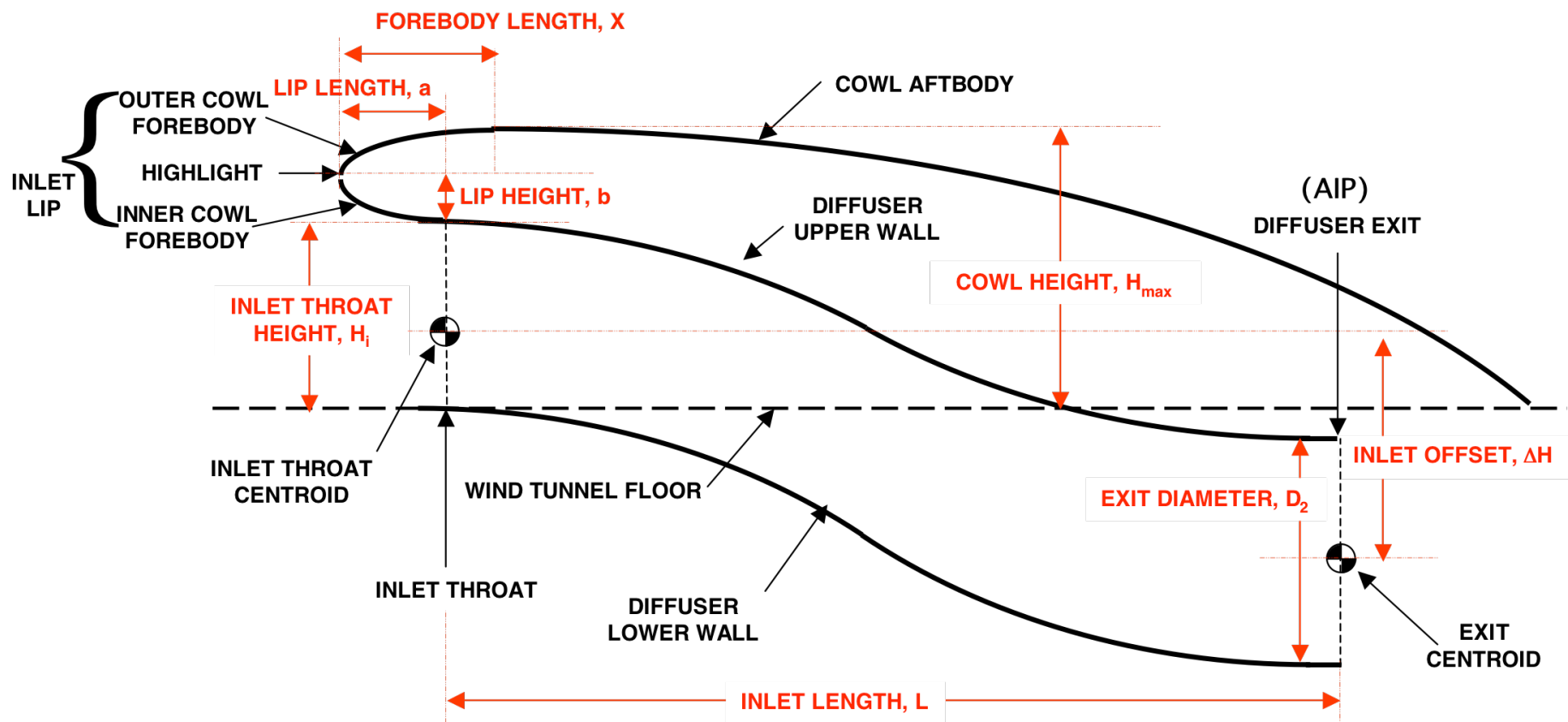

Figure 3. The BLI inlet design parameters shown on a side-view sketch of model centerline.

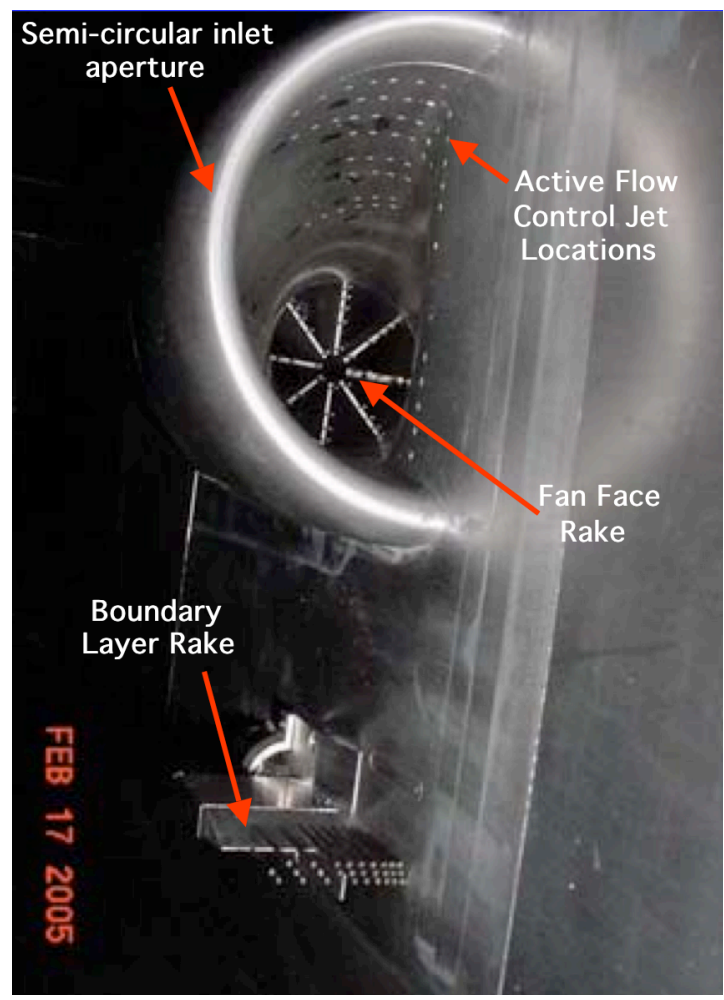

a) Inlet model front view

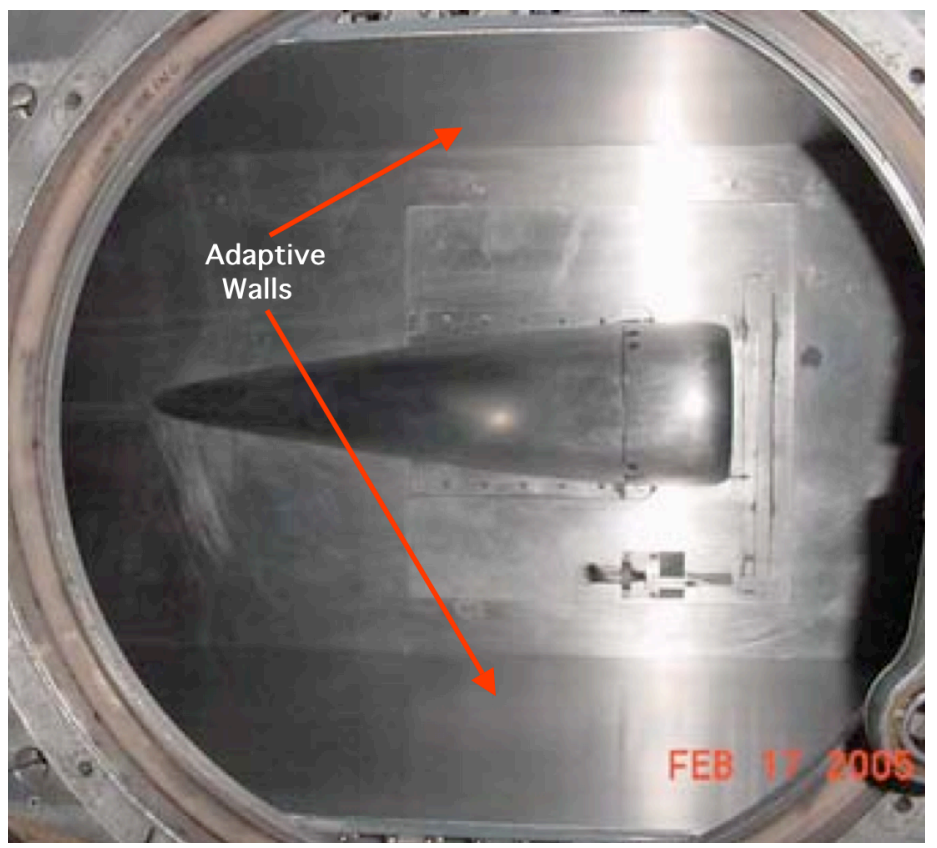

b) Inlet model top view

Figure 4. BLI inlet model as installed in the 0.3-meter TCT test section. 


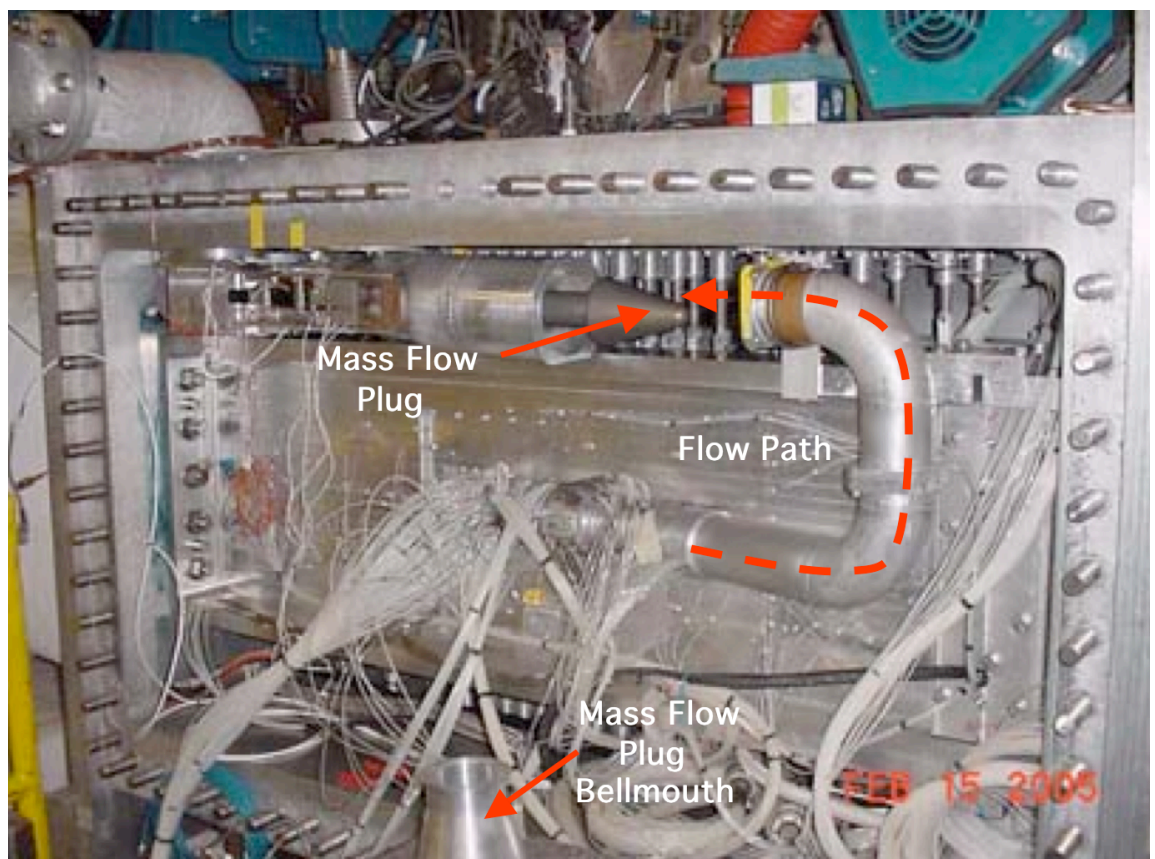

Figure 5. The BLI inlet model mass flow control system assembly providing the flow path out of tunnel test section.

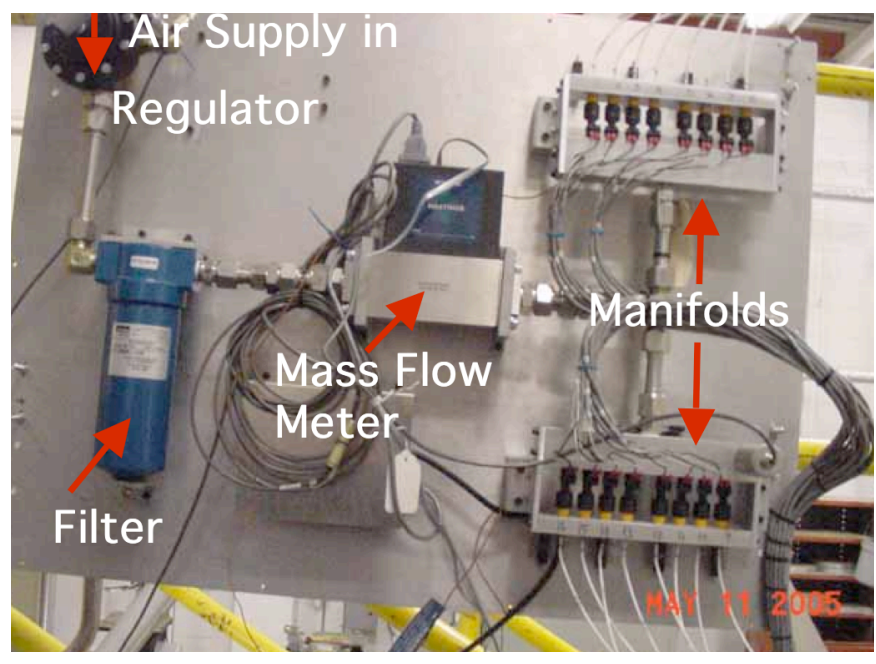

a) Air Supply Panel

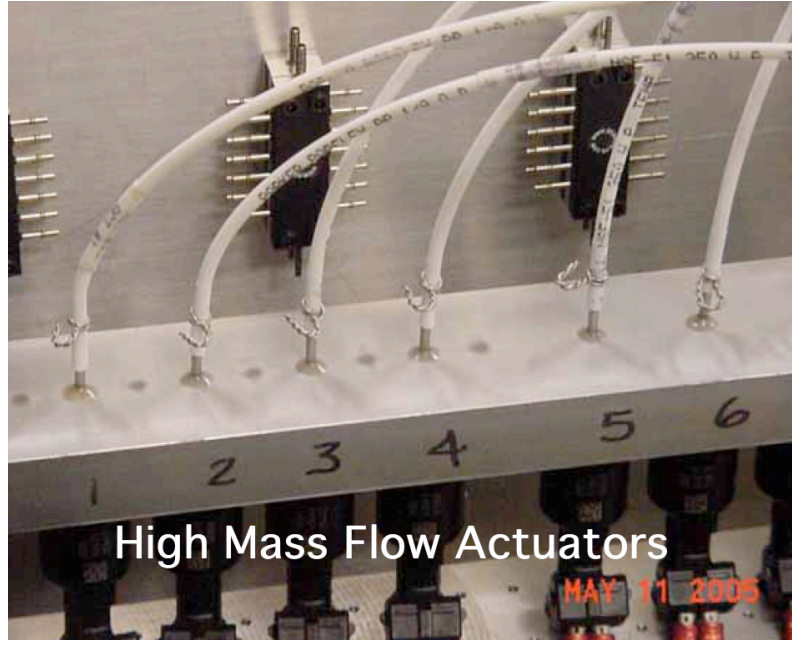

b) Close-up of Actuators in a Manifold

Figure 6. Air supply system used for the inlet model control jet orifices. 


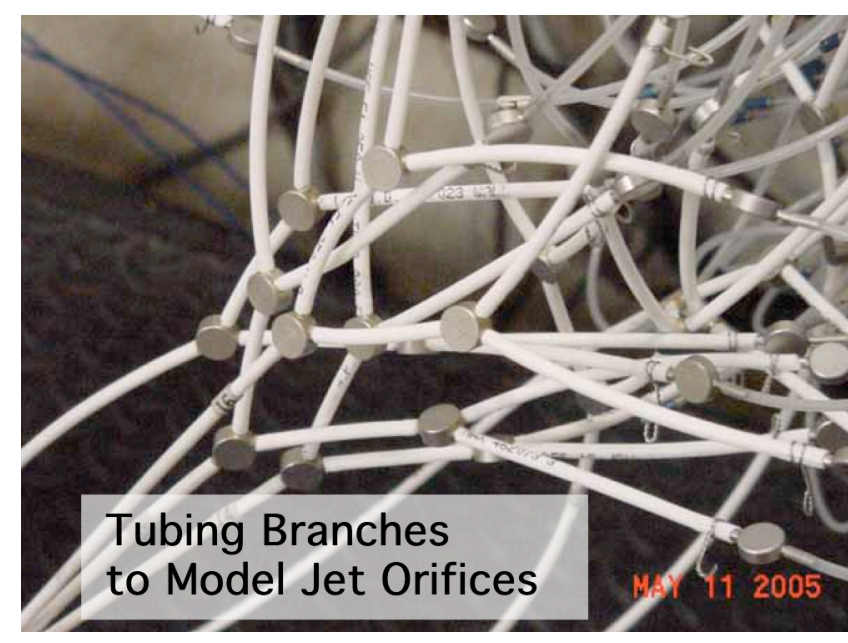

a) Splitting each actuator to multiple jet orifices

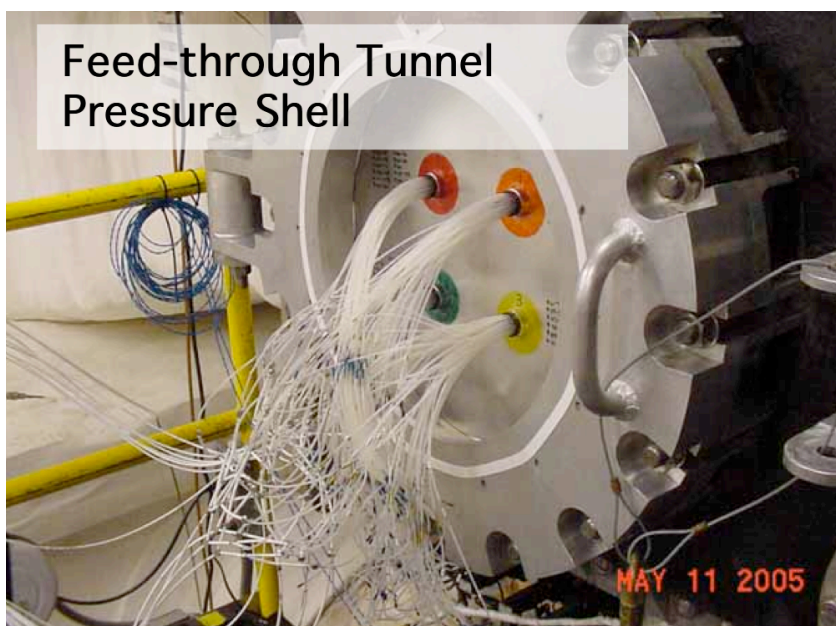

b) Jet tubing entering the tunnel

Figure 7. Control jet supply tubing between the air supply panel and the tunnel pressure shell.

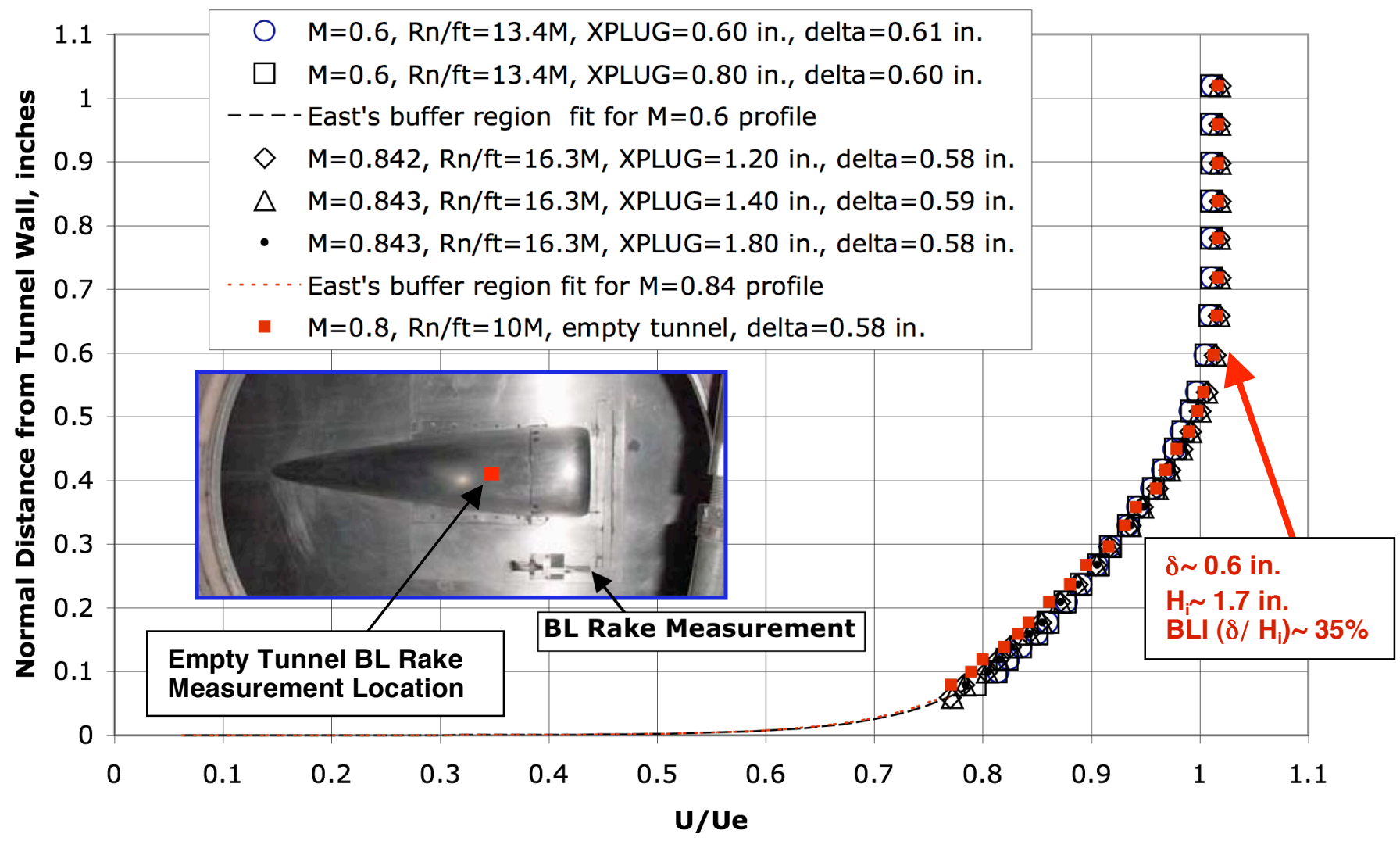

Figure 8. Measured boundary layer profiles in the BLI Inlet experiment. 


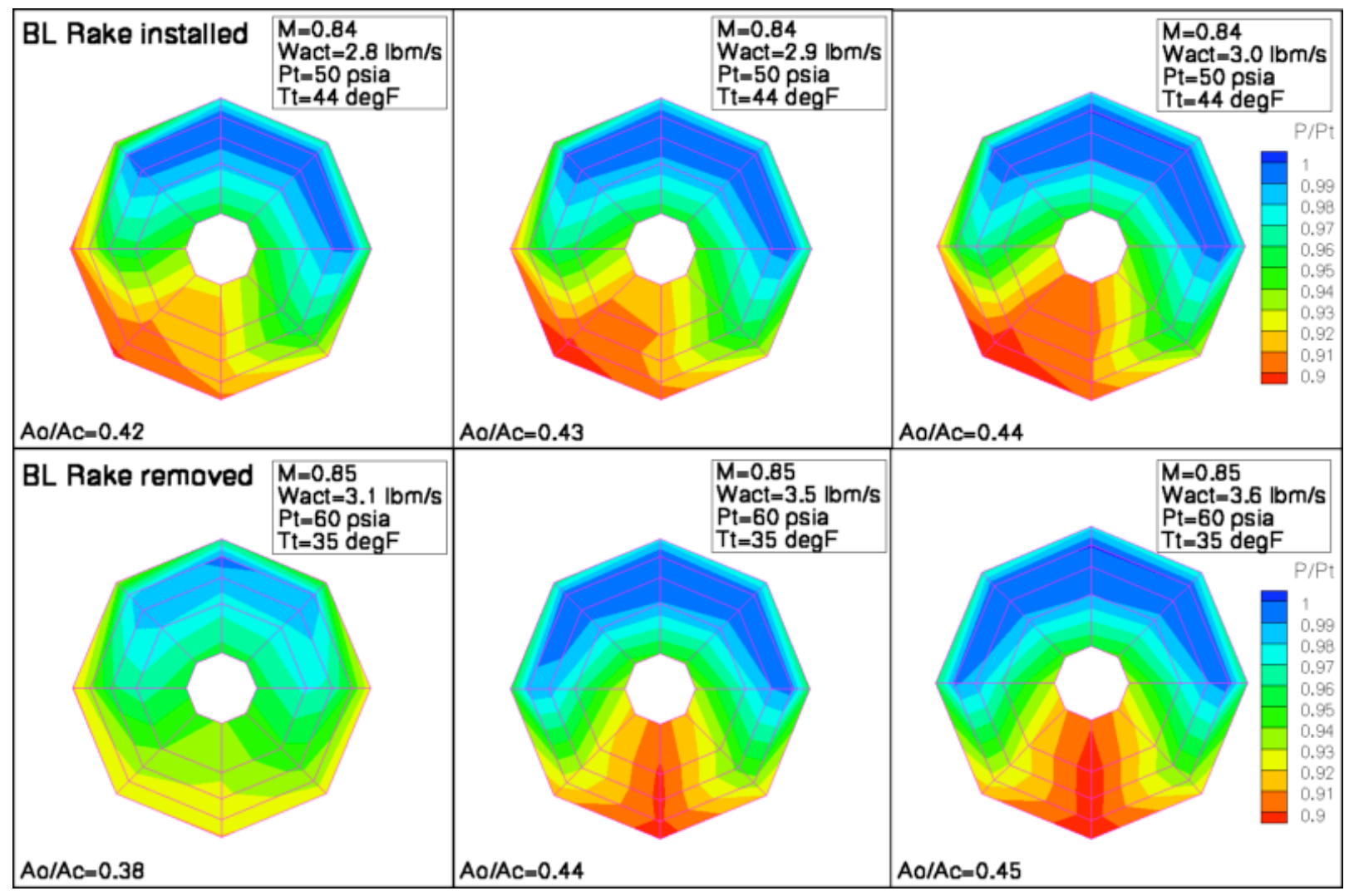

Figure 9. Asymmetric effect of boundary layer rake installation on the measured AIP pressures.

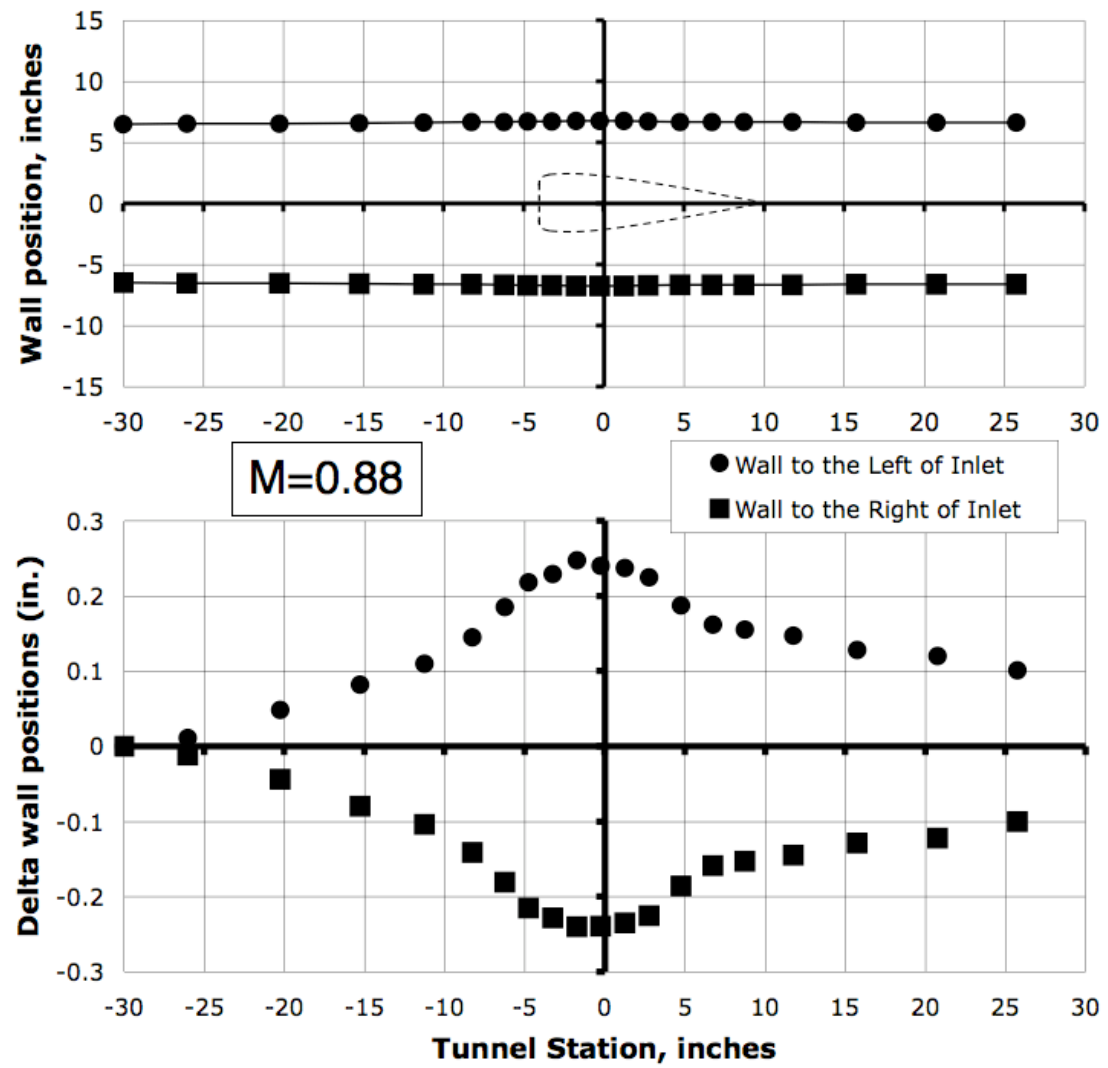

Figure 10. Bottom view of inlet model showing the final adaptive wall positions used throughout the flow control experiment. 


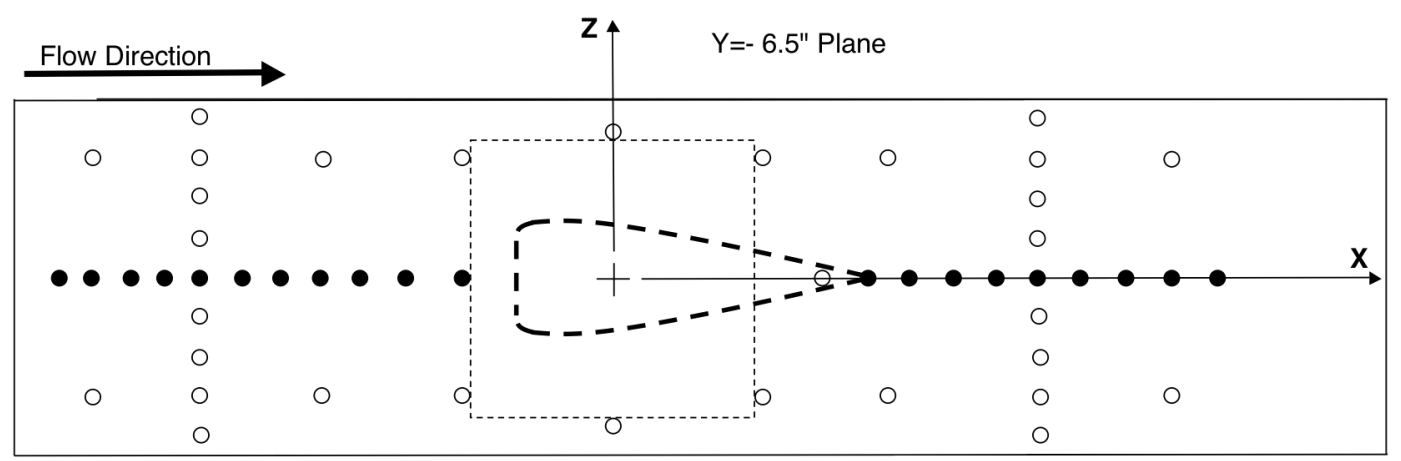

a) tunnel wall inlet model mounts on

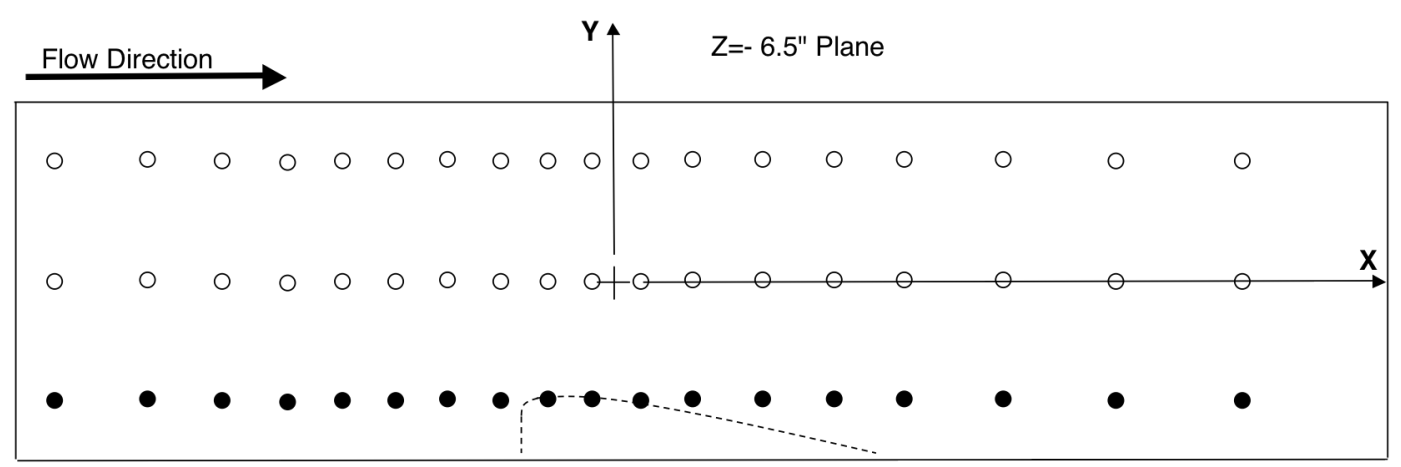

b) tunnel wall on right side of inlet model

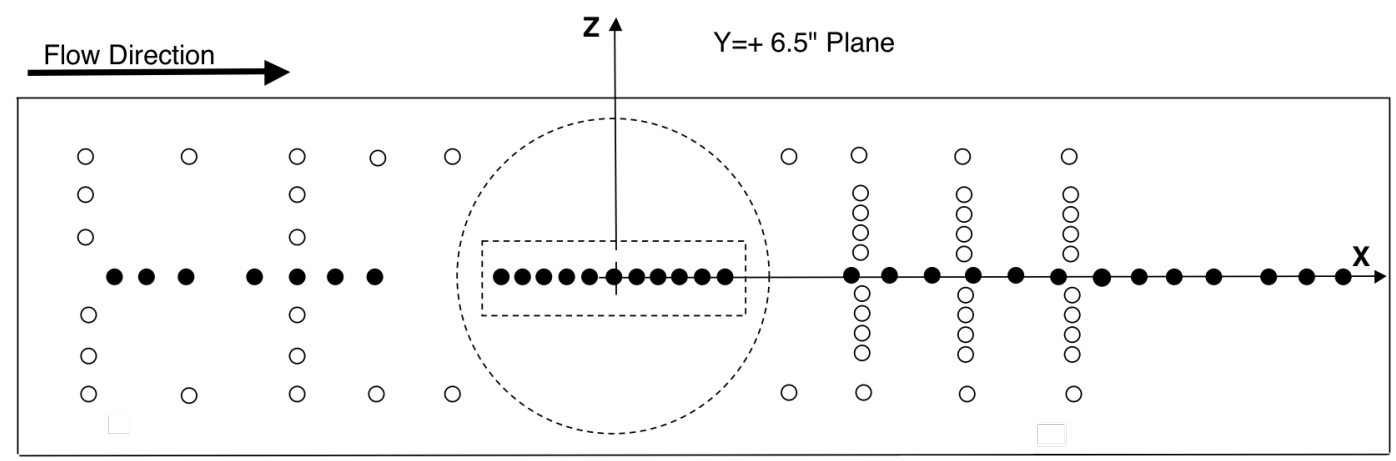

c) tunnel wall opposite of inlet model

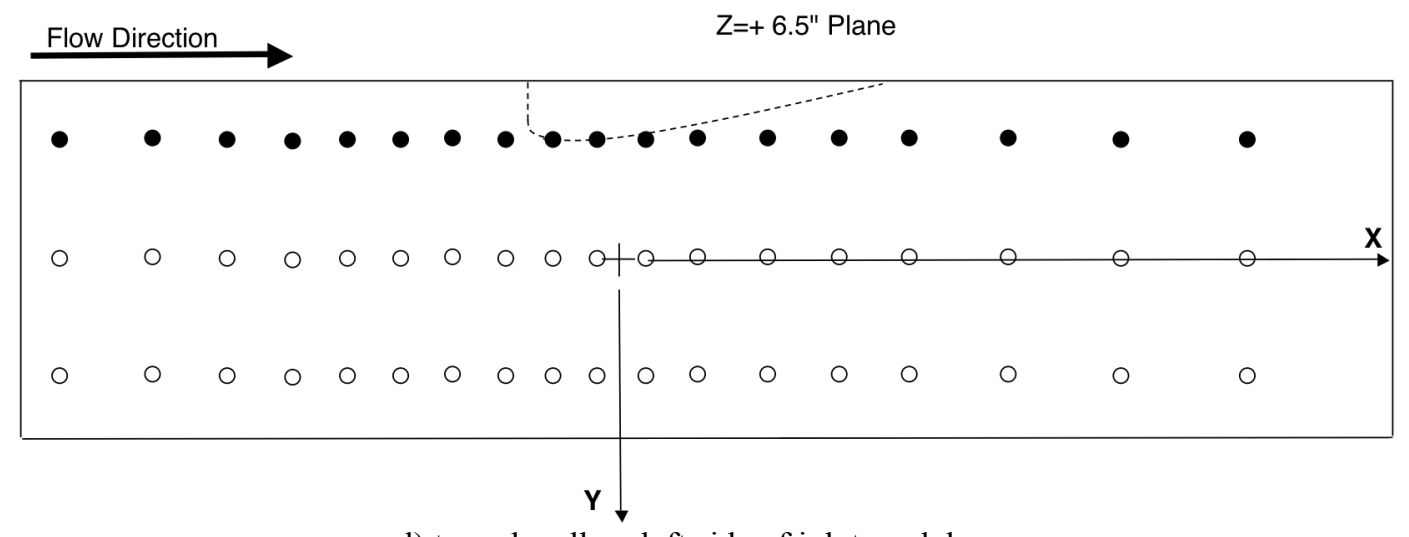

d) tunnel wall on left side of inlet model

Figure 11. Location of tunnel wall pressure orifices used to calculate local Mach number distributions. 


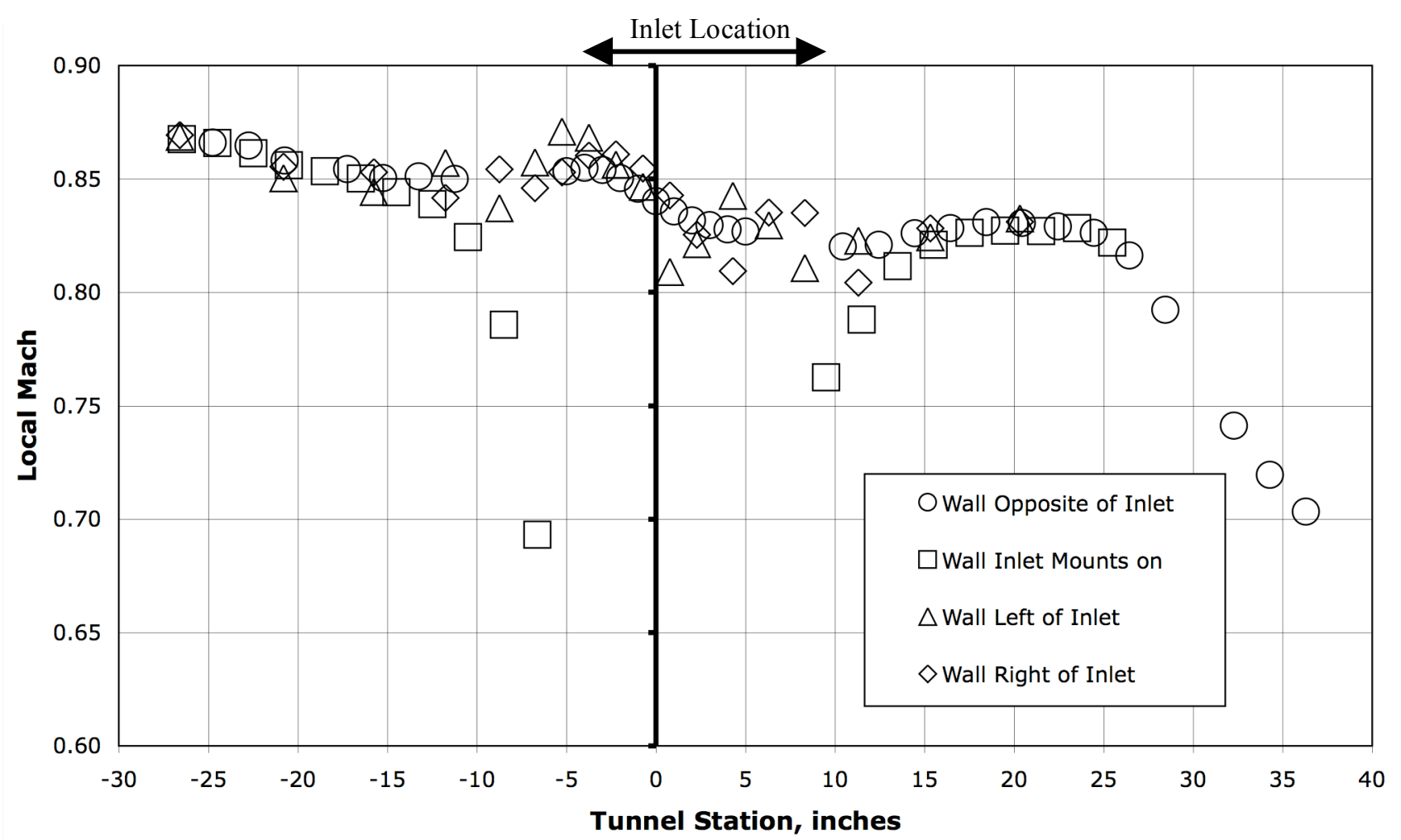

Figure 12. Local Mach distribution from tunnel wall centerline pressure measurements at $M=0.88, P_{t}=30$ psia, $\mathrm{T}_{\mathrm{t}}=\mathbf{8 0}{ }^{\circ} \mathrm{F}, \mathrm{A}_{\mathrm{O}} / \mathrm{A}_{\mathrm{C}}=\mathbf{0 . 5 4}$.

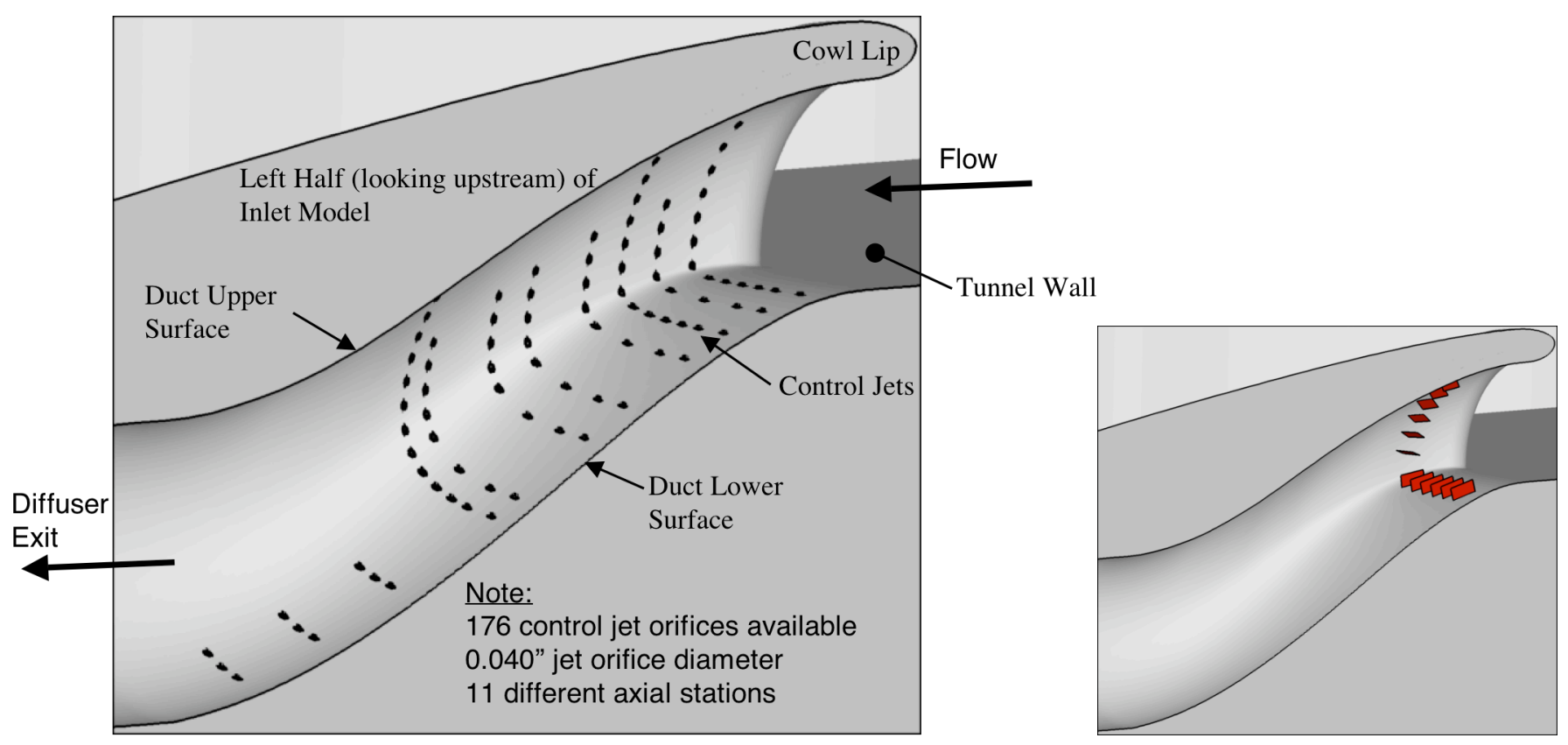

Figure 13. A view of the left (looking upstream) half of the inlet diffuser is shown to illustrate all available jet locations and the general location of the vortex generators when installed. 


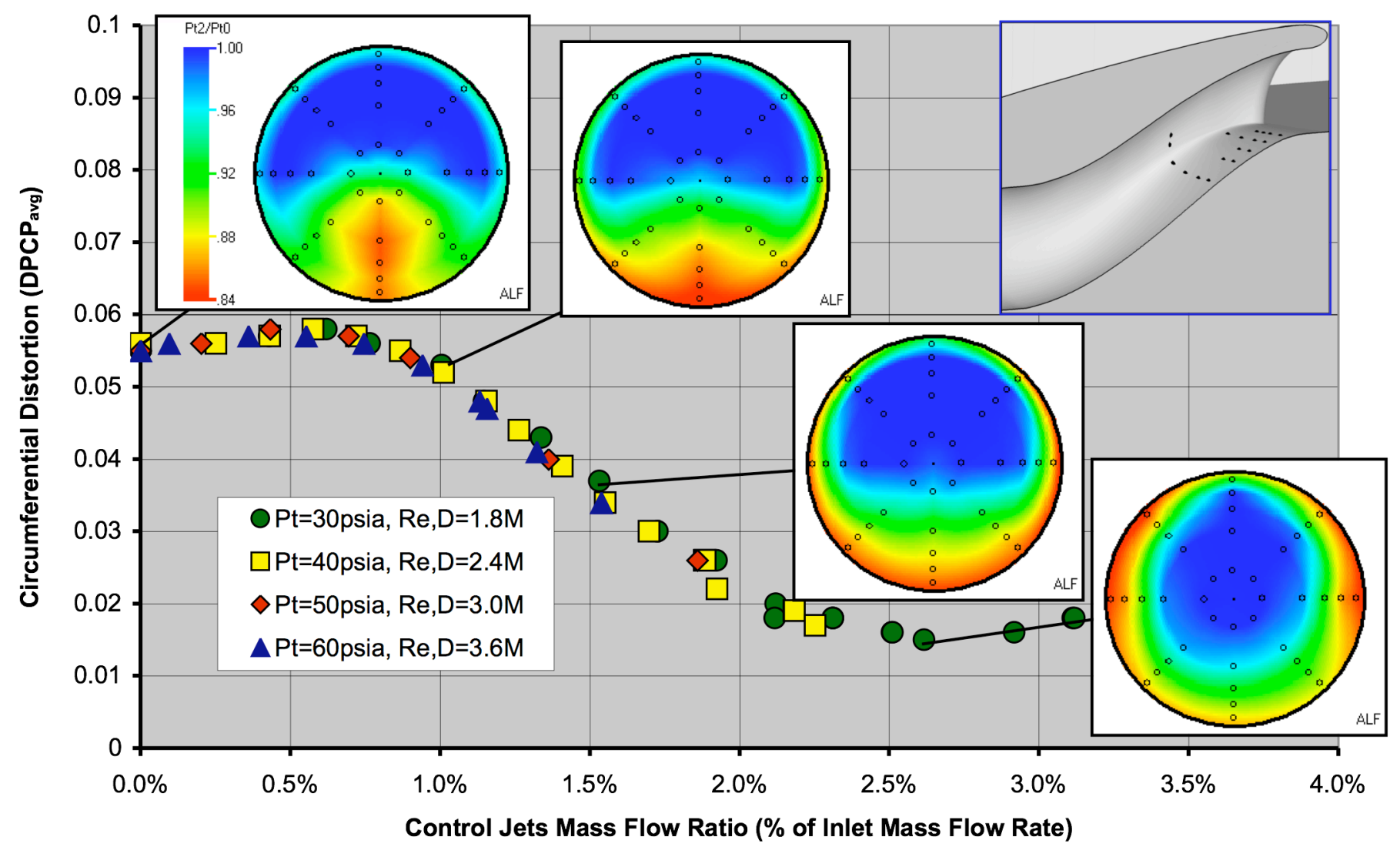

Figure 14. Effects of Tunnel Total Pressure Variation ( $\left.\operatorname{Re}_{D}\right)$ on Distortion Reduction in BLI Inlet Experiment for $M=0.85$, $T_{t}=80^{\circ} \mathrm{F}, A_{0} / A_{C}=0.54$, Configuration $10 \mathrm{w} / 36$ jets.

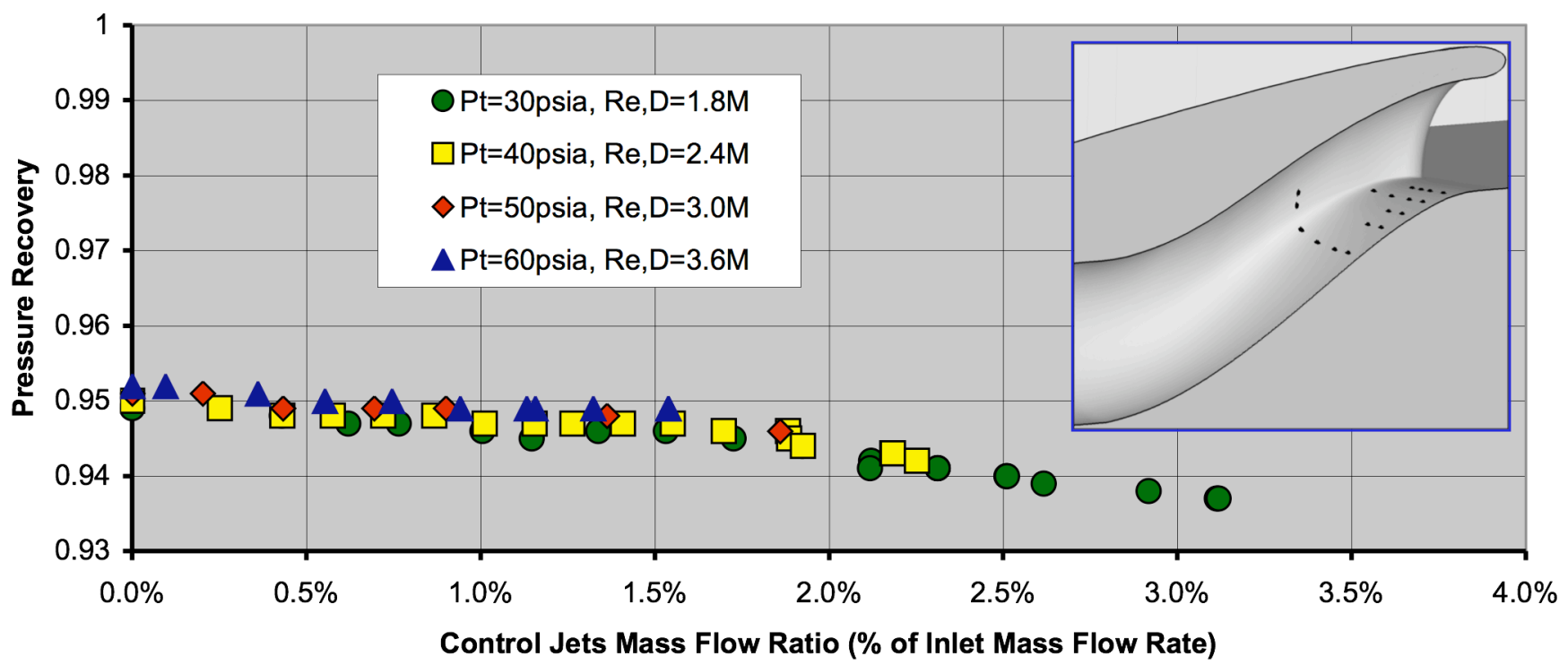

Figure 15. Effects of Tunnel Total Pressure Variation ( $\left.\operatorname{Re}_{\mathrm{D}}\right)$ on Pressure Recovery with AFC in BLI Inlet Test for $M=0.85$, $\mathrm{T}_{\mathrm{t}}=80^{\circ} \mathrm{F}, \mathrm{A}_{\mathrm{O}} / \mathrm{A}_{\mathrm{C}}=0.54$, Config $10 \mathrm{w} / 36$ jets. 




Figure 16. Control Jet Velocity and Distribution Effect on Circumferential Distortion Reduction in BLI Inlet Experiment for $\mathrm{M}=\mathbf{0 . 8 5}, \mathrm{P}_{\mathrm{t}}=30 \mathrm{psia}, \mathrm{T}_{\mathrm{t}}=\mathbf{8 0}{ }^{\circ} \mathrm{F}, \mathrm{A}_{\mathrm{O}} / \mathrm{A}_{\mathrm{C}}=\mathbf{0 . 5 4}$.

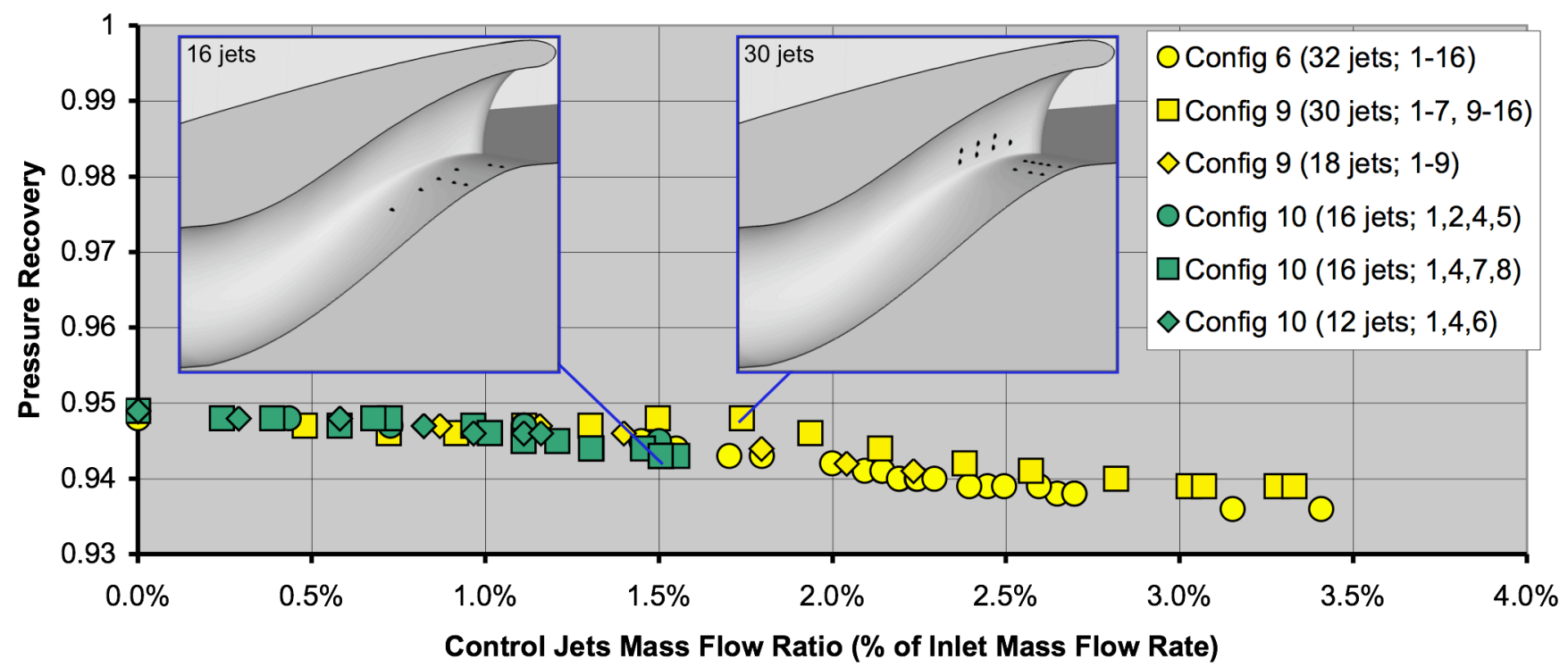

Figure 17. Control Jet Velocity and Distribution Effect on Pressure Recovery in BLI Inlet Experiment for M=0.85, $P_{t}=30$ psia, $T_{t}=80^{\circ} \mathrm{F}, A_{0} / A_{C}=0.54$. 


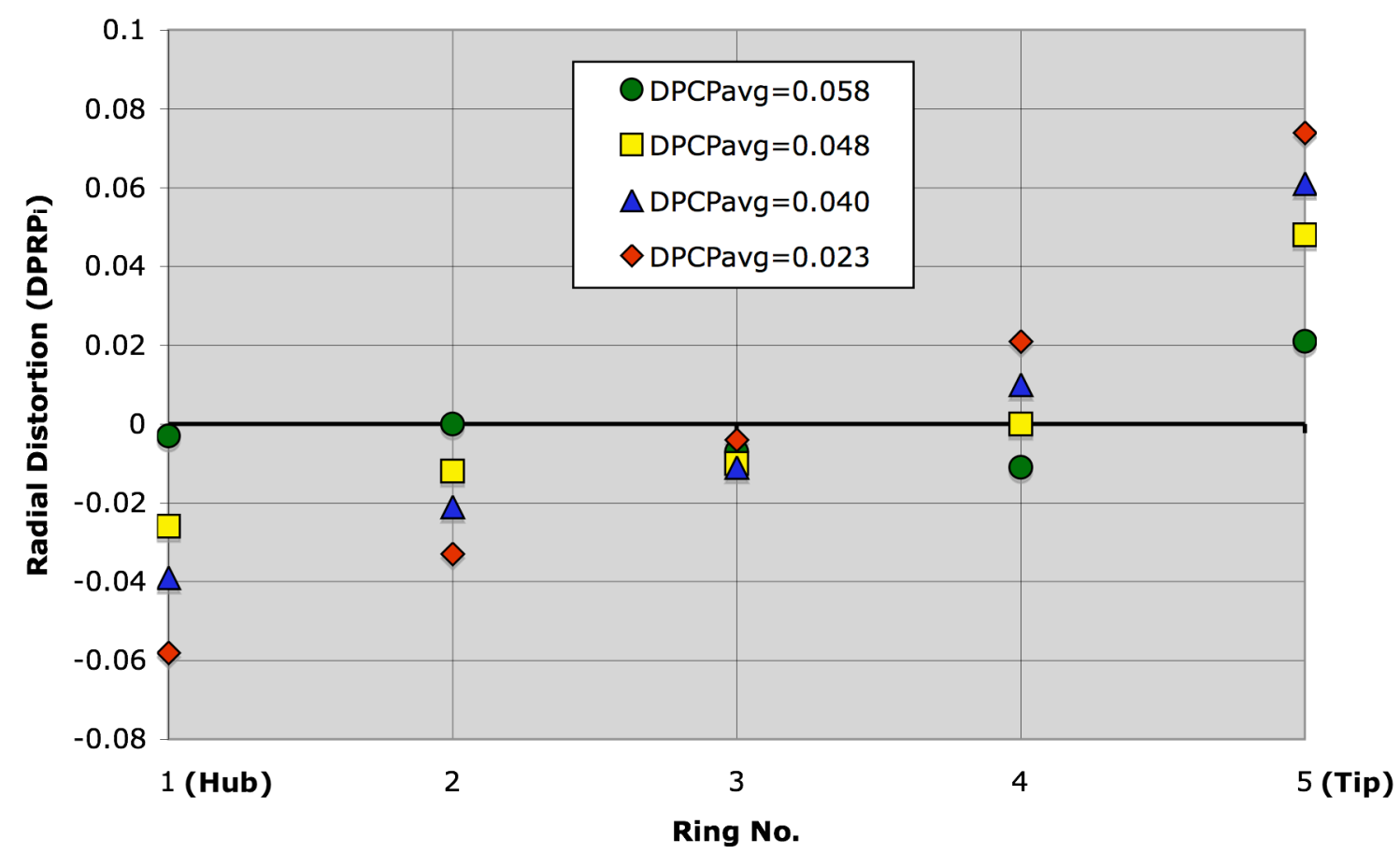

Figure 18. Typical Radial Distortion Character as Control Jets Reduce Circumferential Distortion in BLI Inlet Test for $M=0.85, P_{t}=30$ psia, $T_{t}=80^{\circ} F, A_{0} / A_{C}=0.54$.

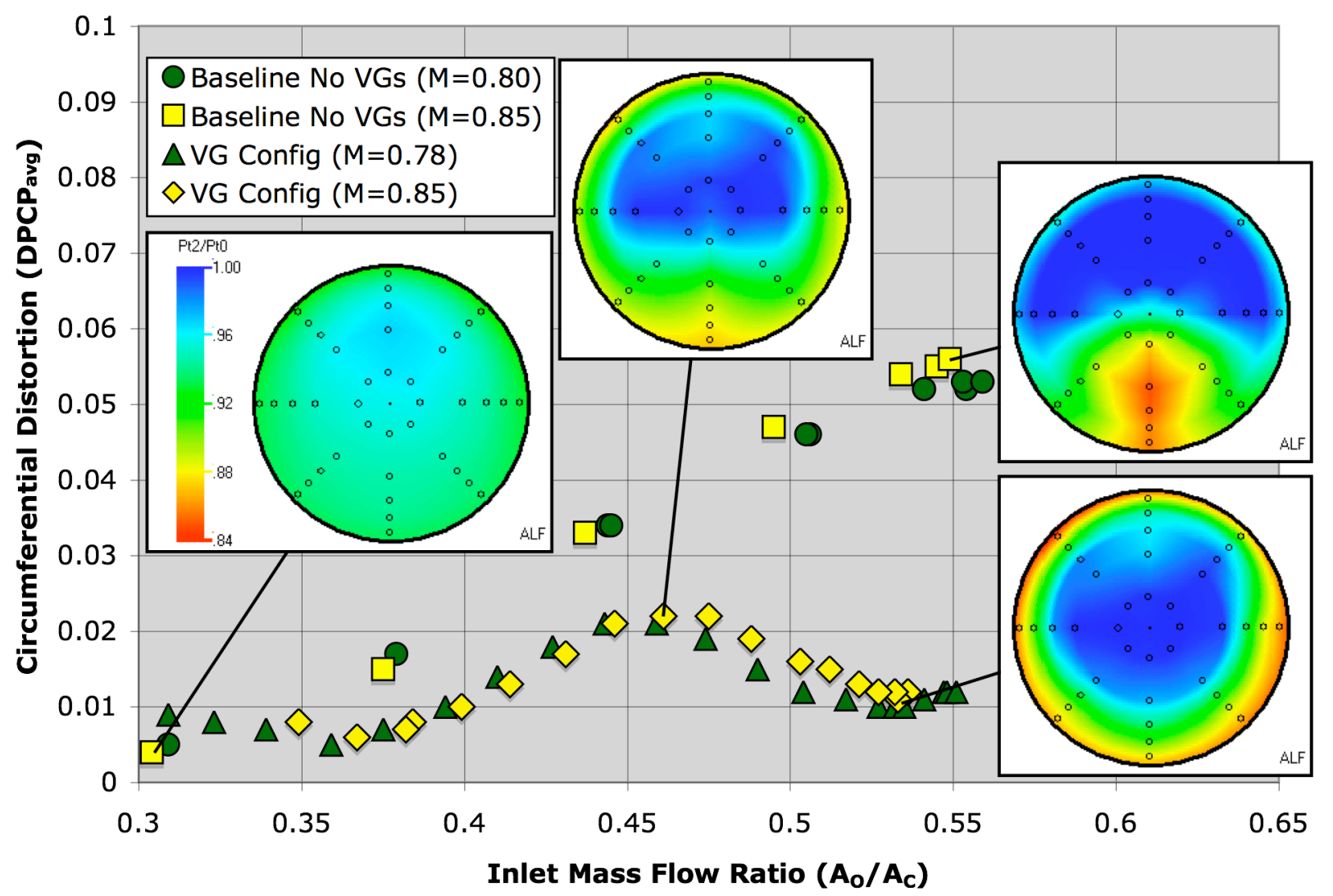

Figure 19. VG Control Effect on Distortion Reduction in BLI Inlet Experiment for $P_{t}=30$ psia, $T_{t}=80^{\circ} F$. 


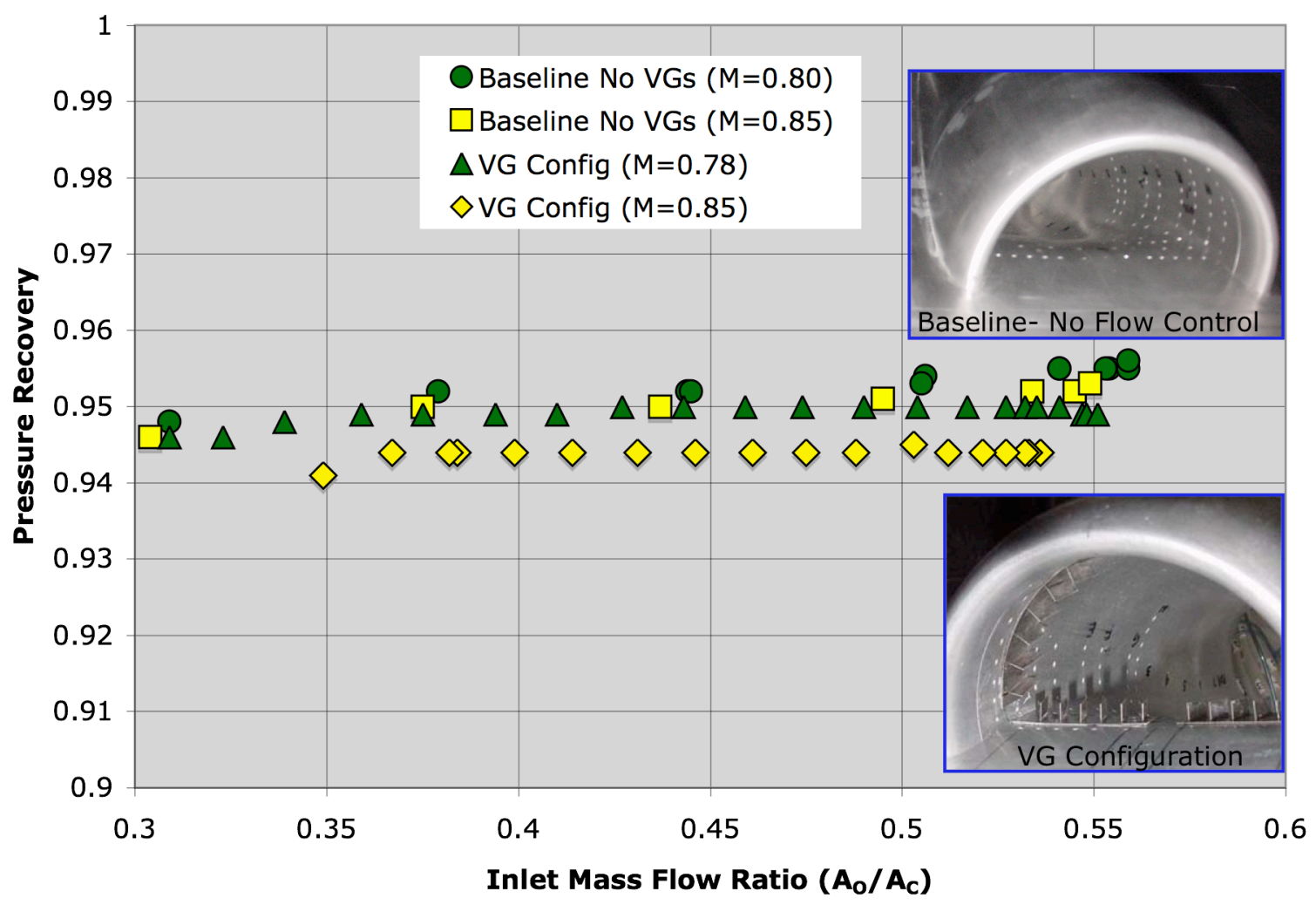

Figure 20. VG Control Effect on Pressure Recovery in BLI Inlet Experiment for $P_{t}=30$ psia, $T_{t}=80^{\circ} F$.

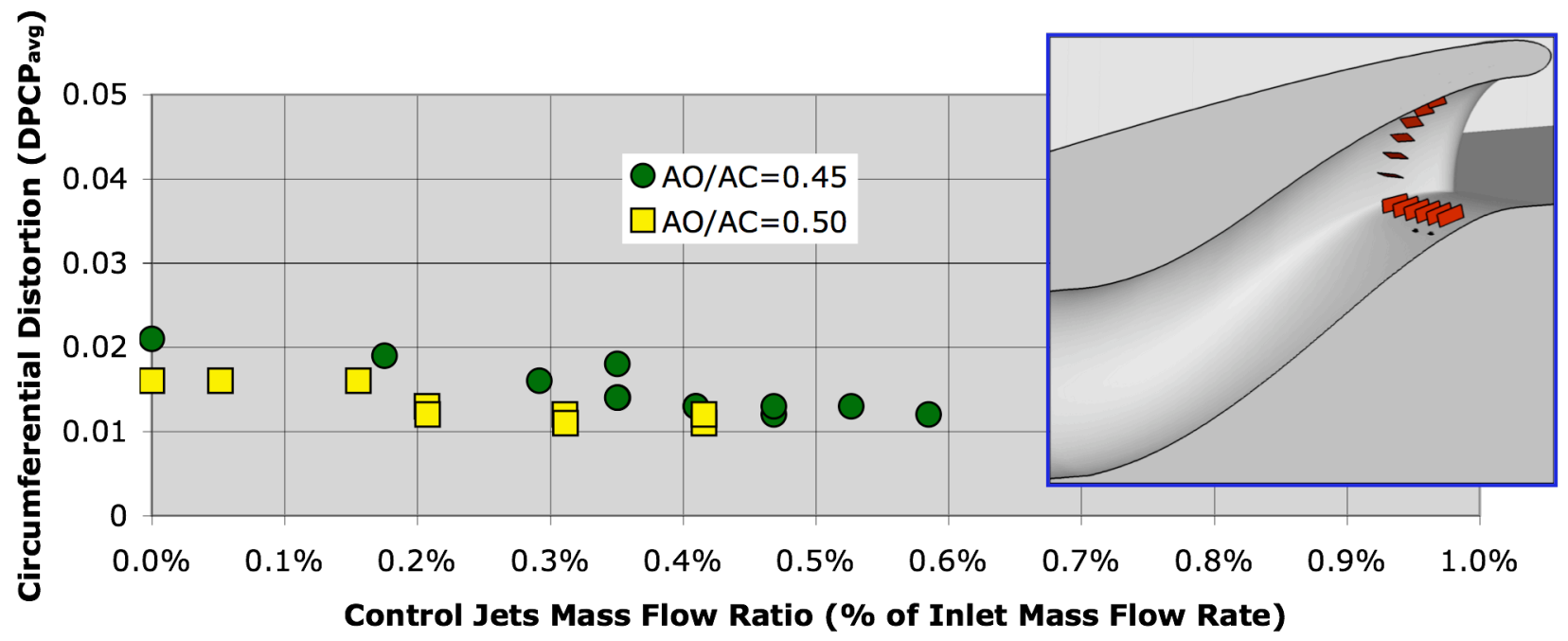

Figure 21. Combined VG and Jet Control Effect on Distortion Reduction in BLI Inlet Test for $M=0.85$, $P_{t}=30$ psia, $\mathrm{T}_{\mathbf{t}}=80^{\circ} \mathrm{F}$, Configuration $11 \mathrm{VGs}$ w/4 Jets. 